\title{
Conjecture principale équivariante, idéaux de Fitting et annulateurs en théorie d'Iwasawa
}

\author{
par THONG NGUYEN QUANG DO
}

\begin{abstract}
RÉSUMÉ. Pour un nombre premier impair $p$ et une extension abélienne $K / k$ de corps de nombres totalement réels, nous utilisons la Conjecture Principale Équivariante démontrée par Ritter et Weiss (modulo la nullité de l'invariant $\mu_{p}$ ) pour calculer l'idéal de Fitting d'un certain module d'Iwasawa sur l'algèbre complète $\mathbb{Z}_{p}\left[\left[G_{\infty}\right]\right]$, où $G_{\infty}=G a l\left(K_{\infty} / k\right)$ et $K_{\infty}$ est la $\mathbb{Z}_{p}$-extension cyclotomique de $K$. Par descente, nous en déduisons la $p$-partie de la version cohomologique de la conjecture de Coates-Sinnott, ainsi qu'une forme faible de la $p$-partie de la conjecture de Brumer
\end{abstract}

ABSTRACT. For an odd prime number $p$ and an abelian extension of totally real number fields $K / k$, we use the Equivariant Main Conjecture proved by Ritter and Weiss (modulo the vanishing of the $\mu_{p}$ invariant) to compute the Fitting ideal of a certain Iwasawa module over the complete group algebra $\mathbb{Z}_{p}\left[\left[G_{\infty}\right]\right]$, where $G_{\infty}=$ Gal $\left(K_{\infty} / k\right), K_{\infty}$ being the cyclotomic $\mathbb{Z}_{p}$-extension of $K$. By descent, this gives the $p$-part of (a cohomological version of) the Coates-Sinnott conjecture, as well as a weak form of the $p$-part of the Brumer conjecture.

\section{Dédié à Georges Gras pour son soixantième anniversaire}

\section{Introduction}

Dans tout cet article, on se placera dans la situation suivante $: K / k$ est une extension galoisienne finie de corps de nombres totalement réels, $p$ un nombre premier impair, $K_{\infty}$ (resp. $k_{\infty}$ ) la $\mathbb{Z}_{p}$-extension cyclotomique de $K(\operatorname{resp} . k), G=\operatorname{Gal}(K / k), \Gamma=\operatorname{Gal}\left(k_{\infty} / k\right), G_{\infty}=\operatorname{Gal}\left(K_{\infty} / k\right)$.

Soit $S$ un ensemble de places de $K$ stable par $G$, contenant les places archimédiennes, les places au-dessus de $p$ et les places qui se ramifient dans l'extension $K / k$. On note $M_{\infty}=M_{\infty}^{S}$ la pro- $p$-extension abélienne $S$-ramifiée (i.e. non ramifiée hors de $S$ ) maximale de $K_{\infty}$, et $X_{\infty}=$ $X_{S}\left(K_{\infty}\right)=\operatorname{Gal}\left(M_{\infty} / K_{\infty}\right)$. On peut dire que l'objectif principal de la 
théorie d'Iwasawa cyclotomique est l'étude de la structure galoisienne du module $X_{\infty}$. Dans le cas où $G=(1)$, la Conjecture Principale classique, qu'on notera $(C P W)$ - démontrée par Mazur \& Wiles [MW1] pour $k=\mathbb{Q}$ et par Wiles [W1] pour tout $k$ totalement réel - donne une interprétation analytique $p$-adique de la série caractéristique de $X_{\infty}$ sur l'algèbre $\Lambda=\mathbb{Z}_{p}[[\Gamma]]$. Pour tenir compte de l'action galoisienne de $G$, une Conjecture Principale Equivariante, ou (CPE), devrait donner, dans le même esprit, une interprétation analytique $p$-adique d'une "série caractéristique équivariante" associée à $X_{\infty}$ sur l'algèbre $\mathbb{A}=\mathbb{Z}_{p}\left[\left[G_{\infty}\right]\right] \ldots$ sauf qu'une telle s.c.e. reste à définir.

Une CPE en termes de complexes parfaits a été proposée par K. Kato ([K], $\S 3$; pour une généralisation, voir e.g. [HK2]) et démontrée récemment dans le cas absolument abélien par Burns et Greither ([BG1]). Cependant, dans le cas cyclotomique étudié ici, il nous semble que le langage des modules reste plus lisible, la structure galoisienne de $X_{\infty}$ pouvant être décrite en termes de $K$-théorie, plus précisément par la suite exacte de localisation reliant $K_{0}$ à $K_{1}$. Une CPE dans ce sens a été proposée par Ritter et Weiss ([RW1 \& 2]) et démontrée par eux récemment dans le cas abélien relatif, en supposant la nullité de l'invariant $\mu_{p}$ du module $X_{\infty}$ : c'est "l'équation $\mathcal{T}$ " de [RW2], thm. 11.

Dans le présent article, on se place dans la même situation que dans [RW2] et l'on se propose d'appliquer leur CPE à la détermination des idéaux de Fitting initiaux de certains $\mathbb{A}$-modules associés à $X_{\infty}$, notamment son morlule "adjoint". Notons que pour tout $\mathbb{A}$-module de torsion $M$ dont la dimension projective est inférieure ou égale à 1 (notation : $p d_{\mathbb{A}} M \leq 1$ ), l'idéal de Fitting inital $F_{\mathbb{A}}(M)$ est principal, donc fournit un bon candidat pour la notion de s.c.e. Mais la dimension projective de $X_{\infty}$ (ou de son adjoint) n'est malheureusement pas finie en général. Heureusement, même non principaux, les idéaux de Fitting gardent suffisamment d'informations arithmétiques pour permettre de démontrer la (version cohomologique de) la conjecture de Coates-Sinnott sur l'annulation galoisienne des groupes de $K$-théorie pairs de l'anneau des entiers de $K$, ainsi qu'une version $p$-adique faible de la conjecture de Brumer sur l'annulation galoisienne du groupe de classes de $K$.

Voici maintenant un plan succint de ce travail : dans un premier temps, nous redémontrons une version (légèrement) améliorée de l'équation $\mathcal{T}$ de Ritter \& Weiss, exprimée en termes d'idéaux de Fitting ( $\S 2)$; dans un second temps, nous utilisons cette CPE pour déterminer l'idéal de Fitting de l'adjoint de $X_{\infty}$, en termes de la pseudo-mesure de Deligne-Ribet attachée au groupe abélien profini $G_{\infty}(\S 3)$; les théorèmes d'annulation galoisienne évoqués plus haut (Coates-Sinnott, Brumer) s'en déduisent par descente $(\S 4)$. 
Nous tenons ici à remercier J. Ritter et A. Weiss pour nous avoir patiemment expliqué les subtilités de [RW1 \& 2]. Il semble que le sujet soit "dans l'air du temps" puisque, dans la période de finalisation de cet article, et notamment aux colloques d'Oberwolfach (février 2002), Caen (juin 2002) et Baltimore (août 2002), nous avons pu prendre connaissance de nombreux travaux indépendants sur le calcul des idéaux de Fitting en théorie d'Iwasawa (des prépublications qui entre-temps sont devenues des publications telles que [BG 2], [G3], [K1], [K2], ...). Le lecteur intéressé trouvera certainement instructif de rapprocher les résultats et comparer les méthodes.

\section{Modules galoisiens}

Rappelons que $\mathbb{A}$ désigne l'algèbre complète $\mathbb{Z}_{p}\left[\left[G_{\infty}\right]\right]$. Tous les $\mathbb{A}$-modules étudiés seront supposés noetheriens. Un tel $\mathbb{A}$-module est de torsion s'il est annulé par un élément central de $\mathbb{A}$ qui ne divise pas zéro.

Le $\mathbb{A}$-module $X_{\infty}=X_{\infty}^{S}$ défini dans l'introduction est de torsion, mais en général, il n'est pas de dimension projective finie. L'idée de départ dans [RW1 \& 2] est de remplacer $X_{\infty}$ par son "enveloppe" $Y_{\infty}$, qui est de dimension projective inférieure ou égale à 1, mais n'est pas de torsion; puis de remplacer $Y_{\infty}$, via la "construction $\Psi$ ", par un quotient $Z_{\infty}$ de $Y_{\infty}$ qui sera à la fois de dimension projective $\leq 1$ et de $\mathbb{A}$-torsion. Notre but, dans cette section préliminaire, va être de redonner une construction de $Y_{\infty}$ par "générateurs et relations", puis de généraliser (légèrement) la construction de $Z_{\infty}$. Cette approche a l'avantage d'être plus fonctorielle que celle de [RW1 \& 2], mais surtout, elle se prêtera mieux au calcul des idéaux de Fitting.

Fixons encore quelques notations. Soit $k_{S}$ (resp. $k_{S}(p)$ ) l'extension algébrique (resp. la pro-p-extension) $S$-ramifiée maximale de $k$, et soit $G_{S}(k)$ (resp. $G_{S}(k)(p)$ ) son groupe de Galois sur $k$. Pour toute extension $L$ de $k$ contenue dans $k_{S}$, on notera $X_{L}=X_{S}(L)=G_{S}^{a b}(L)(p)$ l'abélianisé de $G_{S}(L)(p)$; en particulier, $X_{S}\left(k_{\infty}\right)$ n'est autre que le module $X_{\infty}$ de l'introduction. Comme il s'agit de calculer des idéaux de Fitting, on supposera en outre, à partir de maintenant, que $G_{\infty}$ (donc aussi $G$ ) est abélien. Alors $G_{\infty}$ peut se mettre sous la forme d'un produit direct $G_{\infty}=H \times \widetilde{\Gamma}$, où $H=\operatorname{Gal}\left(K_{\infty} / k_{\infty}\right) \simeq \operatorname{Gal}\left(K \cap k_{\infty} / k\right)$ et $\widetilde{\Gamma}$ est un relèvement de $\Gamma$ dans $G_{\infty}$.

\subsection{Le module $Y_{\infty}=Y_{\infty}^{S}$ :}

Dans cette sous-section, qui est essentiellement de nature homologique, on va rappeler rapidement la construction fonctorielle de "l'enveloppe" $Y_{\infty}$ de $X_{\infty}$ suivant [N1] (voir aussi [J],§ 4 ; [NSW], chap. V, § 4; [RW1], appendix $4 \mathrm{~A})$. 
Soit $F$ le sous-corps de $K$ fixé par le $p$-Sylow $P$ de $G$. Posons $Q=$ Gal $(F / k)$ et $\mathcal{G}=G a l\left(F_{S}(p) / k\right)$. Alors $\mathcal{G}$ est un groupe profini de type fini, qu'on peut présenter sous la forme $\mathcal{G} \simeq \mathcal{F} / \mathcal{W}$, où $\mathcal{F}$ est pro-libre sur $d$ générateurs. De plus, $c d_{p} \mathcal{G}=c d_{p}\left(G_{S}(F)(p)\right)$ car $p$ ne divise pas l'ordre de $Q$, et il est connu que $c d_{p}\left(G_{S}(F)(p)\right) \leq 2$ puisque $p$ est impair. Pour toute extension galoisienne $L / k$, contenant $F$ et contenue dans $F_{S}(p)$, notons $J=G a l(L / k) \simeq \mathcal{F} / \mathcal{R}, R_{L}^{a b}=R^{a b}(J)$ le pro-p-quotient maximal de $\mathcal{R}^{a b}$ (c'est "le" (pro)-p-module des relations de $J$ ) et $W^{a b}$ le pro-p-quotient maximal de $\mathcal{W}^{a b}$. Comme $c d_{p} \mathcal{G} \leq 2$, on sait que $W^{a b}$ est projectif sur l'algèbre complète $\mathbb{Z}_{p}[[\mathcal{G}]]$. On désignera, pour simplifier, par $\mathbb{Z}_{p} J$ l'algèbre de groupe $\mathbb{Z}_{p}[J]$ ou l'algèbre complète $\mathbb{Z}_{p}[[J]]$ suivant que $J$ est fini ou profini, et par $\Delta J$ (resp. $\Delta \mathcal{G})$ l'idéal d'augmentation de $\mathbb{Z}_{p} J$ (resp. $\left.\mathbb{Z}_{p}[[\mathcal{G}]]\right)$. Alors, en suivant exactement les arguments de [N1], $\S 1$, on obtient un diagramme commutatif exact :
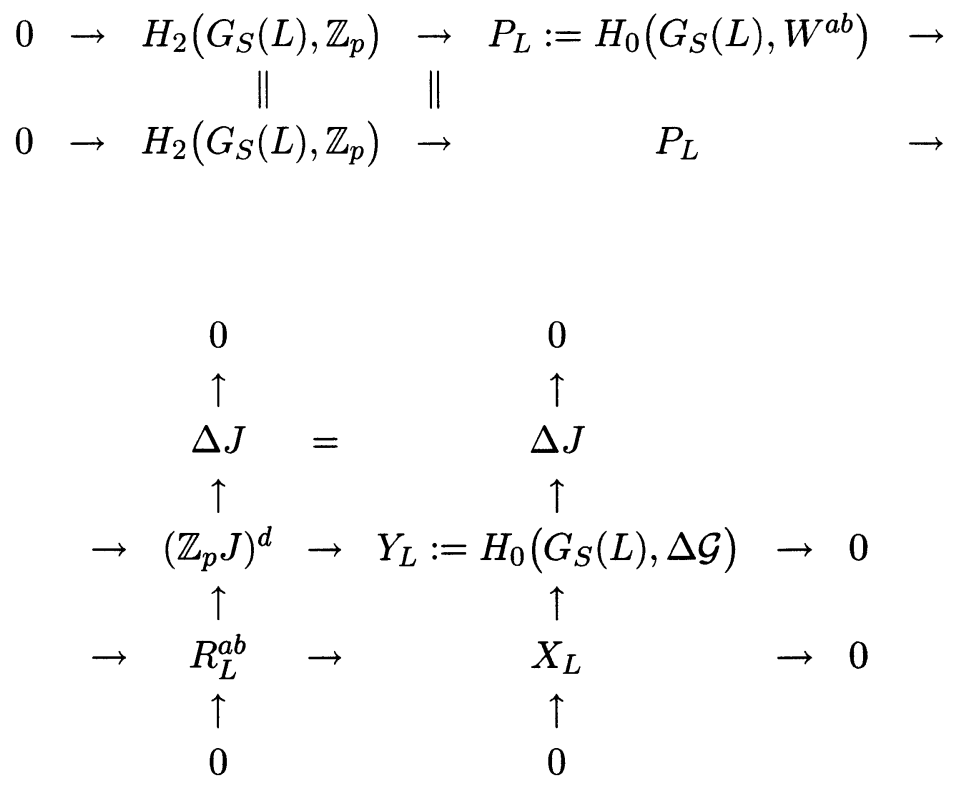

\subsubsection{Commentaires :}

i) Si l'on savait (ce qui n'est pas le cas, voir e.g. [NSW], p. 532) que le groupe profini $G_{S}(k)$ est de type fini, les arguments précédents s'appliqueraient à $G_{S}(k)$ en lieu et place de $\mathcal{G}$, et les résultats seraient à la fois plus généraux et plus simples à démontrer.

ii) Le groupe $H_{2}\left(G_{S}(L), \mathbb{Z}_{p}\right)$ est le défaut de la conjecture de Leopoldt pour $L$ et $p$, c'est-à-dire que $L$ vérifie la conjecture de Leopoldt (usuelle si $J$ est fini, "faible" si $J$ est infini) si et seulement si $H_{2}\left(G_{S}(L), \mathbb{Z}_{p}\right)=0$. 
iii) La première colonne exacte est la pro- $p$-version de la résolution de Lyndon, qui montre en particulier que $R^{a b}(J)$ est un invariant de $J$ à un $\mathbb{Z}_{p} J$-facteur projectif près.

iv) Comme $W^{a b}$ est $\mathbb{Z}_{p}[[\mathcal{G}]]$-projectif, $P_{L}$ est a priori $\mathbb{Z}_{p} J$-projectif, mais en fait, dans les hypothèses de 1.1 et si $L$ vérifie la conjecture de Leopoldt (usuelle ou faible), $P_{L}$ est libre de rang $(d-1)$. En effet, tous les modules qui interviennent dans le diagramme (1) sont sur l'algèbre $\mathbb{Z}_{p}[Q][[J(p)]]$, où $J(p)$ est le pro-p-groupe $G a l(L / F)$. Comme $Q$ est d'ordre premier à $p$, on peut faire le découpage habituel suivant les idempotents primitifs $e_{\chi}$ associés aux caractères $p$-adiques $\chi$ de $\Delta$ (à conjugaison près), et obtenir des diagrammes commutatifs exacts $\left(1_{\chi}\right)$ sur les algèbres $A_{\chi}:=\mathbb{Z}_{p}[\chi][[J(p)]]$. Comme $J(p)$ est un pro-p-groupe, $A_{\chi}$ est une algèbre locale, le module projectif $e_{\chi} P_{L}$ est en fait libre, et la première ligne de $\left(1_{\chi}\right)$ s'écrit : $0 \rightarrow$ $A_{\chi}^{r_{\chi}} \rightarrow A_{\chi}^{d} \rightarrow e_{\chi} Y_{L} \rightarrow 0$. Mais comme $J(p)$ est un pro-p-groupe, le même raisonnement exactement que dans [N1], propos. 1.7, montre que $-1+$ $d-r_{\chi}$ est la caractéristique d'Euler-Poincaré de $G_{S}(F)(p)$, c'est-à-dire zéro puisque $F$ est totalement réel. On a donc $e_{\chi} P_{L} \simeq A_{\chi}^{d-1}$, et par suite $P_{L} \simeq\left(\mathbb{Z}_{p} J\right)^{d-1}$.

En considérant le cas particulier $L=K_{\infty}$, et sachant que la $\mathbb{Z}_{p}$-extension cyclotomique $K_{\infty}$ vérifie automatiquement la conjecture faible de Leopoldt ([N1], § 2), on obtient :

1.1.2. Proposition : Si $K / k$ est une extension abélienne de corps de nombres totalement réels, on a un diagramme commutatif exact naturel :

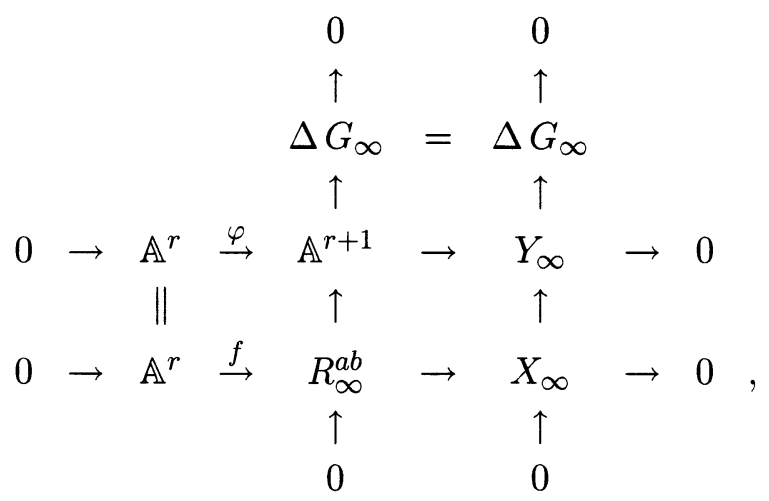

où $\mathbb{A}=\mathbb{Z}_{p}\left[\left[G_{\infty}\right]\right], X_{\infty}=X_{K_{\infty}}, Y_{\infty}=Y_{K_{\infty}}, R_{\infty}^{a b}=R_{K_{\infty}}^{a b}$.

\subsection{Les modules $Z_{\infty}$ et $z_{\infty}$ :}

Rappelons maintenant rapidement la "construction $\Psi$ " de Ritter \& Weiss, qui permet de remplacer $Y_{\infty}$ par un module de torsion. Soit $\Delta G_{\infty}$ l'idéal d'augmentation de $\mathbb{A}=\mathbb{Z}_{p}\left[\left[G_{\infty}\right]\right]$. 
Pour tout élément $d_{\infty} \in \Delta G_{\infty}$, non diviseur de zéro dans $\mathbb{A}$, choisissons une pré-image arbitraire $y_{\infty} \in Y_{\infty}$ (voir la colonne de droite dans le diagramme (2)). En posant $\psi(1)=d_{\infty}$ et $\Psi(1)=y_{\infty}$, on définit des homomorphismes $\psi$ et $\Psi$ qui prennent place dans un diagramme commutatif

$$
\begin{array}{rll}
0 & & 0 \\
\downarrow & \downarrow \\
\mathbb{A} & =\mathbb{A} \\
& \downarrow \Psi & \\
& \downarrow \psi \\
0 \rightarrow X_{\infty} \rightarrow Y_{\infty} & \rightarrow \Delta G_{\infty} \rightarrow 0
\end{array}
$$

et qui sont injectifs car $d_{\infty}$ n'est pas diviseur de zéro. On obtient ainsi des A-modules de torsion $Z_{\infty}:=$ coker $\Psi$ et $z_{\infty}:=$ coker $\psi$ tels que la suite $0 \rightarrow X_{\infty} \rightarrow Z_{\infty} \rightarrow z_{\infty} \rightarrow 0$ est exacte. De plus, le $\mathbb{A}$-module $Z_{\infty}$ est de dimension projective inférieure ou égale à 1 , ce qu'on écrira : $p d_{\mathbb{A}} Z_{\infty} \leq 1$. (NB : ces notations cachent la dépendance par rapport à $d_{\infty}$ )

Par "pullback", on obtient un diagramme commutatif exact, où l'application $\varphi$ est comme dans (2) :

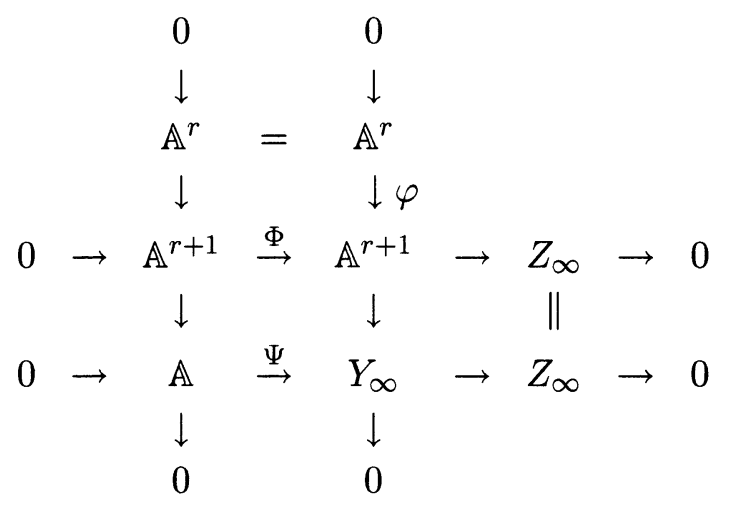

Le jeu dans la suite va consister à calculer l'idéal de Fitting initial de $Z_{\infty}$, soit $F_{\AA} Z_{\infty}=(\operatorname{dét} \Phi)(\operatorname{voir} \S 3)$.

\section{La CPE revisitée}

On garde les hypothèses du début, c'est-à-dire que $K / k$ est une extension abélienne de corps de nombres totalement réels. "L'équation $\mathcal{T}$ " de Ritter $\&$ Weiss est une certaine Conjecture Principale Equivariante qui relie un objet algébrique à un objet analytique : 


\subsection{L'objet algébrique :}

La construction de l'objet algébrique au niveau infini, noté $\mho_{S}$ dans [RW $1 \&$ 2], imite la construction de "l'invariant $\Omega$ relevé" (qui est lui-même une version équivariante de l'invariant $\Omega$ de Chinburg) au niveau fini. Il s'agit essentiellement de modifier $Z_{\infty}$ pour se débarrasser de la dépendance par rapport au choix de $d_{\infty}$. Le lemme suivant généralise légèrement la construction de Ritter \& Weiss. Fixons d'abord quelques notations :

$$
H=\operatorname{Gal}\left(K_{\infty} / k_{\infty}\right), e=\frac{1}{|H|} \sum_{h \in H} h \quad \text { (c'est l'idempotent de } \mathbb{Z}_{p}[H]
$$

associé au caractère trivial de $H), \Gamma=\langle\gamma\rangle, \widetilde{\Gamma}=\langle\widetilde{\gamma}\rangle=$ un relèvement de Gal $\left(k_{\infty} / k\right)$ dans $G_{\infty}\left(\operatorname{donc} G_{\infty} \simeq H \times \widetilde{\Gamma}\right)$.

$Q \mathbb{A}=$ l'anneau total des fractions de $\mathbb{A}$, i.e. le localisé de $\mathbb{A}$ par rapport au système multiplicatif formé des non-diviseurs de zéro.

$\mathcal{M}=$ l'ordre maximal de $\mathbb{A}$, i.e. la fermeture intégrale de $\mathbb{A}$ dans $Q \mathbb{A}$.

Alors :

\subsubsection{Lemme :}

i) Le noyau de l'augmentation $\mathcal{M} \rightarrow \mathbb{Z}_{p}$ est un idéal principal, engendré par l'élément $b_{\infty}:=(\widetilde{\gamma}-1) e+(1-e)$.

ii) Tout élément $d_{\infty} \in \Delta G_{\infty}$, non diviseur de zéro, peut s'écrire sous la forme $d_{\infty}=b_{\infty} c_{\infty}$, avec $c_{\infty} \in(Q \mathbb{A})^{\times}$.

\section{Preuve :}

L'assertion i) est évidente, par découpage suivant les idempotents orthogonaux de $H$ dans $Q \mathbb{A}$. Pour ii), il suffit de remarquer que si $d_{\infty}$ n'est pas diviseur de zéro, $c_{\infty}$ n'est pas non plus diviseur de zéro, donc est inversible dans $Q \mathbb{A}$.

À partir des définitions et de 2.1.1 ii), on peut montrer comme dans [RW1], propos. 4.6, que l'élément $\mho_{S}:=\left[Z_{\infty}\right]-\partial\left[Q \mathbb{A}, c_{\infty}\right] \in K_{0} T(\mathbb{A})$, où $\partial$ est l'homomorphisme de connexion $K_{1}(Q \mathbb{A}) \rightarrow K_{0} T(\mathbb{A})$, ne dépend pas de $d_{\infty}$.

\subsection{L'objet analytique :}

Dans toute la suite, $\chi$ désignera un caractère d'ordre fini $\operatorname{de} G_{\infty}$ à valeurs dans $\overline{\mathbb{Q}}_{p}^{\times}$. On rappelle qu'à tout $\chi$ est associée une série de Deligne-Ribet $G_{\chi, S}(T) \in \mathbb{Z}_{p}[\chi][[T]]$, à partir de laquelle on peut définir la fonction $L_{p, S}(1-s, \chi)$ (c'est la fonction $L p$-adique privée de facteurs euleriens en $S$, normalisée comme dans [W1]) par la formule : $L_{p, S}(1-s, \chi)=$ $G_{\chi, S}\left(u^{s}-1\right) / H_{\chi}\left(u^{s}-1\right)$. Ici, l'on a fixé un générateur topologique de 
$\Gamma=\operatorname{Gal}\left(k_{\infty} / k\right), u=\kappa(\gamma)-1$, où $\kappa$ est le caractère cyclotomique de $\Gamma$, et

$$
H_{\chi}(T)=\left\{\begin{array}{lll}
\chi(\gamma)(1+T)-1 & \text { si } & \chi_{/ H}=1 \\
1 & \text { si } & \chi_{/ H} \neq 1
\end{array} \quad(\text { type } W)\right.
$$

D'après [RW1], propos. 5.4, il existe un unique élément $\Theta_{S} \in \mathcal{M}$ tel que $\chi\left(\Theta_{S}\right)=G_{\chi, S}(0)$ pour tout caractère $\chi$ d'ordre fini de $G_{\infty}$. Plus précisément, $\Theta_{S}:=\sum_{\psi \in \widehat{H}} G_{\psi, S}(\widetilde{\gamma}-1) e_{\psi}$, la somme portant sur les caractères $\psi$ de $H$ (considérés comme caractères de $G_{\infty}$ triviaux sur $\widetilde{\Gamma}$ ) et $e_{\psi}$ désignant l'idempotent associé à $\psi$. En fait, $\Theta_{S}$ est inversible dans $Q \mathbb{A}$, du fait de la non-nullité de chaque série $G_{\psi, S}(T)$.

La Conjecture Principale classique (ou théorème de Wiles) peut alors s'énoncer :

(CPW) Il existe une unité $\varepsilon \in \mathcal{M}^{\times}$telle que $\partial\left(\varepsilon \Theta_{S}\right)=\mho_{S}$ (voir [RW2], thm. 6 a)).

La Conjecture Principale équivariante selon Ritter \& Weiss (ou équation $\mathcal{T})$ se lit :

(CPE1) $\quad \partial\left(\Theta_{S}\right)=\mho_{S} \quad$ (voir l'introduction de [RW2]),

et elle est démontrée dans [RW2], $\S 6$, modulo la nullité de l'invariant $\mu_{p}$ attaché au module d'Iwasawa $X_{\infty}$. Notons que $\mu_{p}=0$ dépend seulement de $p$, pas de $S$. Il résulte immédiatement des définitions que l'équation $\mathcal{T}$ est équivalente à l'égalité suivante entre idéaux de $\mathbb{A}$ :

(CPE2) $\quad F_{\mathbb{A}}\left(Z_{\infty}\right)=\left(c_{\infty} \Theta_{S}\right)$, où $Z_{\infty}=\operatorname{coker} \Psi$ et $d_{\infty}=b_{\infty} c_{\infty}($ voir

Cette formulation a l'avantage (dans notre optique) de faire intervenir les idéaux de Fitting, mais le désavantage de cacher l'indépendance par rapport au choix de $d_{\infty}$ (ou de $c_{\infty}$ ). On se propose de donner une démonstration simplifiée (par rapport à [RW2]) de la CPE2. Notons que l'expression de cette CPE2 sous-entend que $c_{\infty} \Theta_{S} \in \mathbb{A}$, ce qui n'est pas évident a priori. L'explication réside dans les propriétés de la pseudo-mesure de DeligneRibet :

\subsubsection{Rappels (voir [Se]) :}

Pour tout groupe profini commutatif $A$ ayant un quotient isomorphe à $\mathbb{Z}_{p}$, une pseudo-mesure $p$-adique sur $A$ est un élément $\lambda \in Q\left(\mathbb{Z}_{p}[[A]]\right)$ tel que $(\sigma-1) \lambda$ soit une mesure, i.e. un élément de $\mathbb{Z}_{p}[[A]]$, pour tout $\sigma \in A$. Pour $A=X_{S}(k)$ (notations du $\S 1$ ), le théorème de Deligne-Ribet ([DR] ; voir le compte-rendu de [Se]) donne l'existence d'une unique pseudo-mesure $\lambda_{S}$ sur $X_{S}(k)$ telle que, pour tout $s \in \mathbb{Z}_{p}$, pour tout caractère $\chi$ d'ordre fini de $X_{S}(k)$, on ait $L_{p, S}(1-s, \chi)=\left\langle\chi \kappa^{s}, \lambda_{S}\right\rangle$. Ici $L_{p, S}$ est relative à $k, \kappa$ 
désigne le complément du caractère de Teichmüller $\omega$ dans la décomposition habituelle du caractère cyclotomique, $\chi_{c y c}=\omega \kappa$, et $\langle.,$.$\rangle est l'intégration$ par rapport à une pseudo-mesure ([Se], 1.12).

Notons alors $\theta_{S} \in Q \mathbb{A}$ l'image de $\lambda_{S}$ par le passage au quotient $X_{S}(k) \rightarrow$ $G_{\infty}$, au sens suivant : pour tout choix d'un relèvement $\hat{\gamma}$ de $\tilde{\gamma}$ dans $X_{S}(k)$, l'élément $(\hat{\gamma}-1) \lambda_{S}$ appartient à $\mathbb{Z}_{p}\left[\left[X_{S}(k)\right]\right]$, et l'on désigne par $Z_{S}$ son image dans $\mathbb{Z}_{p}\left[\left[G_{\infty}\right]\right]=\mathbb{A} ;$ par définition, $\theta_{S}=(\widetilde{\gamma}-1)^{-1} Z_{S} \in Q \mathbb{A}:$ c'est la pseudo-mesure de Deligne-Ribet associée à $G_{\infty}$.

\subsubsection{Lemme :}

Pour $d_{\infty}=b_{\infty} c_{\infty}$ comme dans le lemme 2.1.1 ii), on a : $c_{\infty} \Theta_{S}=$ $d_{\infty} \theta_{S} \in \mathbb{A}$.

\section{Preuve :}

Pour simplifier les notations, posons $T=\tilde{\gamma}-1$ et notons $\left\langle\chi_{1}, \chi_{2}\right\rangle$ le produit scalaire de deux caractères. L'élément $Z_{S} \in \mathbb{A}=\Lambda[H]$ précédement introduit s'écrit de façon unique : $Z_{S}=\sum_{h \in H} Z_{S}(h, T) h$, avec $Z_{S}(h, T) \in \Lambda=$ $\mathbb{Z}_{p}[[T]]$. La formule suivante ([RW2], propos. 12) est alors une conséquence directe de la caractérisation de la pseudo-mesure de Deligne-Ribet :

$$
G_{\psi, S}(T) / T^{\langle\psi, 1\rangle}=\sum_{h \in H} \psi(h) Z_{S}(h, T) / T \text {, pour tout caractère } \psi \text { de } H
$$

(considéré comme caractère de $G_{\infty}$ ).

$$
\text { Or } \Theta_{S} / b_{\infty}=\Theta_{S} /(\widetilde{\gamma}-1) e+(1-e)=\sum_{\psi \in \widehat{H}} \frac{G_{\psi, S}(T)}{T^{\langle\psi, 1\rangle}} e_{\psi} \text {, où } e_{\psi} \text { est }
$$

l'idempotent usuel associé à $\psi$. Donc $\Theta_{S} / b_{\infty}=\theta_{S}$, et $c_{\infty} \Theta_{S}=d_{\infty} \theta_{S}$. De plus, $d_{\infty} \theta_{S} \in \mathbb{A}$ car $d_{\infty} \in \Delta G_{\infty}$ et $\theta_{S}$ est une pseudo-mesure sur $G_{\infty}$.

La CPE à démontrer est donc équivalente à l'égalité :

$$
(C P E 3) \quad F_{\mathbb{A}}\left(Z_{\infty}\right)=\left(d_{\infty} \theta_{S}\right)
$$

\subsubsection{Théorème : ([RW2], thm. 11).}

Pour toute extension abélienne $K / k$ de corps totalement réels telle que $\mu_{p}=0$, les $C P E i$ sont vraies $(i=1,2,3)$.

\section{Preuve :}

Comme $p d_{\mathbb{A}}\left(Z_{\infty}\right) \leq 1$, l'idéal de Fitting $F_{\mathbb{A}}\left(Z_{\infty}\right)$ est principal, et il s'agit de montrer l'égalité de deux idéaux principaux de $\mathbb{A}$. Or deux tels idéaux coïncident si et seulement leurs localisés coïncident en tout idéal premier de hauteur 1 : c'est une propriété générale des anneaux de Cohen-Macaulay ; pour une démonstration directe dans le cas spécifique de l'algèbre $\mathbb{A}$, voir e.g. [BG 1], lemme 6.3. Le découpage habituel suivant les idempotents 
orthognaux de la non- $p$-partie de $H$ permet immédiatement de se ramener au cas où $H$ est un $p$-groupe. Les idéaux premiers $\mathcal{P}$ de hauteur 1 de $\mathbb{A}=\Lambda[H]$ sont alors de deux types :

i) l'unique idéal premier $\mathcal{P}_{s}$ de hauteur 1 contenant $p$, appelé singulier. Il est facile de voir que $\mathcal{P}_{s}=(p, \Delta H)$ et que $\mathbb{A}_{\mathcal{P}_{s}}=\Lambda_{(p)}[H]$

ii) les idéaux premiers $\mathcal{P}$ de hauteur 1 ne contenant pas $p$, appelés réguliers, et pour lesquels les localisés $\mathbb{A}_{\mathcal{P}}$ sont des anneaux de valuation discrète.

Localisons donc les deux membres de (CPE3) :

a) En $\mathcal{P} \neq \mathcal{P}_{s}, p$ est inversible et les idéaux de Fitting sont tous principaux. La suite exacte $0 \rightarrow X_{\infty} \rightarrow Z_{\infty} \rightarrow z_{\infty} \rightarrow 0$ localisée en $\mathcal{P}$ donne, en notant $F($. $)$ les idéaux de Fitting sur $\mathbb{A}_{\mathcal{P}}: F\left(Z_{\infty}\right)_{\mathcal{P}}=F\left(X_{\infty}\right)_{\mathcal{P}} \cdot F\left(z_{\infty}\right)_{\mathcal{P}}$

Or, par construction, on a une suite exacte $0 \rightarrow z_{\infty} \rightarrow \mathbb{A} / d_{\infty} \mathbb{A} \rightarrow \mathbb{Z}_{p} \rightarrow$ 0 , d'où $F\left(z_{\infty}\right)_{\mathcal{P}}=\left(d_{\infty}\right)_{\mathcal{P}} \cdot F\left(\mathbb{Z}_{p}\right)_{\mathcal{P}}^{-1}=\left(d_{\infty}\right)_{\mathcal{P}}\left(b_{\infty}\right)_{\mathcal{P}}^{-1}$. L'égalité à démontrer revient donc à l'égalité $F\left(X_{\infty}\right)_{\mathcal{P}}=\left(b_{\infty} \mathcal{O}_{S}\right)_{\mathcal{P}}$, ou encore $F\left(X_{\infty}\right)_{\mathcal{P}}=\left(\Theta_{S}\right)_{\mathcal{P}}$.

Puisque $p$ est inversible dans $\mathbb{A}_{\mathcal{P}}$, on est dans la situation semi-simple et l'égalité cherchée dans $\mathbb{A}_{\mathcal{P}}$ est en fait à démontrer dans $\mathcal{M}_{\mathcal{P}}$. Compte tenu de l'égalité des invariants $\mu_{p}$ algébrique et analytique dans $\mathcal{M}$ ([RW1], fin du $\S 5.2$ ), ce n'est rien d'autre que la Conjecture Principale classique (théorème de Wiles) localisée en $\mathcal{P}$.

b) En $\mathcal{P}_{s}$, on a besoin de l'hypothèse supplémentaire $\mu_{p}=0$. Comme la CPE1 est indépendante de $d_{\infty}$, on peut choisir $d_{\infty} \in \Delta G_{\infty}$ tel que $d_{\infty} \notin \mathcal{P}_{s}$ (par exemple, $d_{\infty}=\widetilde{\gamma}-1$ ). Alors $\left[z_{\infty}\right]_{\mathcal{P}_{s}}=0$ car $d_{\infty}$ est inversible dans $\mathbb{A}_{\mathcal{P}_{s}}$, donc $\left[Z_{\infty}\right]_{\mathcal{P}_{s}}=\left[X_{\infty}\right]_{\mathcal{P}_{s}}=0$ car $\mu_{p}=0$. On a rappelé plus haut (voir $(\mathrm{CPW}))$ que la Conjecture Principale classique s'écrit $F_{\mathbb{A}}\left(Z_{\infty}\right)=\left(\varepsilon d_{\infty} \theta_{S}\right)$, où $\varepsilon \in \mathcal{M}^{\times}$, et $\varepsilon \in \mathbb{A}_{\mathcal{P}}^{\times}$pour tout $\mathcal{P} \neq \mathcal{P}_{s}$ d'après a). La nullité de $\left[Z_{\infty}\right]_{\mathcal{P}_{s}}$ signifie que $\varepsilon d_{\infty} \theta_{S}$, donc aussi $\varepsilon$, est inversible dans $\mathbb{A}_{\mathcal{P}_{s}}$.

En résumé, on a montré que $\varepsilon \in \mathbb{A}^{\times}$, c'est-à-dire que la CPE1 est vraie, donc aussi toutes les conjectures $\mathrm{CPE} \mathrm{i}, \mathrm{i}=1,2,3$.

\subsection{Formulaire :}

On rassemble ici, pour un usage ultérieur, diverses formules sur $\Theta_{S}$ et $\theta_{S}$ et leurs liens mutuels. On rappelle que $T:=\widetilde{\gamma}-1$.

i) Par définition, $\Theta_{S}=\sum_{\psi \in \widehat{H}} G_{\psi, S}(T) e_{\psi}$, et d'après 2.2.2, $\theta_{S}=\Theta_{S} / b_{\infty}$, avec $b_{\infty}=T e+(1-e), e=e_{1}$.

ii) Le développement de $\theta_{S}$ suivant les caractères de $H$ peut s'écrire :

$$
\theta_{S}=\frac{G_{1, S}(T)}{T} e+\sum_{\substack{\psi \in \hat{H} \\ \psi \neq 1}} G_{\psi, S}(T) e_{\psi}
$$


Or $\quad G_{1, S}(T)=G_{1, S}(0)+T G_{1, S}^{*}(T)$, avec $G_{1, S}(0)=\prod_{\substack{v \in S \\ v \nvdash p}}\left(1-(N v)^{-1}\right)$.

En posant $R_{S}=G_{1, S}(0)$, on peut séparer la "partie polaire" et la "partie principale" de $\theta_{S}$ :

$$
\theta_{S}=\frac{R_{S}}{T} e+G_{1, S}^{*}(T) e+\sum_{\substack{\psi \in \widehat{H} \\ \psi \neq 1}} G_{\psi, S}(T) e_{\psi}
$$

iii) On peut aussi développer $T \theta_{S}$ "suivant $H$ " dans $\Lambda[H]$ :

$$
T \theta_{S}:=Z_{S}(T)=\sum_{h \in H} Z_{S}(h, T) h,
$$

avec $Z_{S}(h, T) \in \Lambda$ pour tout $h \in H$.

En écrivant $Z_{S}(h, T)=Z_{S}(h, 0)+T Z_{S}^{*}(h, T)$ et en posant $Z_{S}^{*}(T):=$ $\sum_{h \in H} Z_{S}^{*}(h, T) h$, on obtient l'écriture canonique de la pseudo-mesure $\theta_{S}$ ([Se], 1-15) :

$$
\theta_{S}=\frac{R_{S}}{|H|} \nu_{H} \frac{1}{T}+Z_{S}^{*}(T)
$$

avec $\frac{R_{S}}{|H|} \in \mathbb{Z}_{p}$ et $\nu_{H}=\sum_{h \in H} h=$ la mesure de Haar de $H$ (convenablement normalisée).

Le lien avec l'écriture de ii) est donné par la formule $T G_{\psi, S}(T)=$ $T^{\langle\psi, 1\rangle} \sum_{h \in H} \psi(h) Z_{S}(h, T)$ pour tout caractère $\psi \neq 1$ de $H$.

\section{Idéaux de Fitting sur $\mathbb{A}$}

La CPE3 précédemment démontrée donne une interprétation $p$-adique analytique de $F_{\mathbb{A}}\left(Z_{\infty}\right)=$ (dét $(\Phi)$ ) (voir le diagramme (3)), l'élément dét $(\Phi) \in \mathbb{A}$ jouant le rôle de "série caractéristique équivariante". Malheureusement, l'objet principal, le module $X_{\infty}$, n'est pas en général de dimension projective finie sur $\mathbb{A}$, et l'on n'en connaît pas de résolution projective. La recette pour tourner cette difficulté est bien connue en théorie "homotopique" des modules (voir e.g. [J], ou [NSW], chap. V) : elle consiste à introduire le foncteur $M \rightarrow M^{+}=H_{0} m_{\mathbb{A}}(M, \mathbb{A})$ et ses foncteurs dérivés $M \rightarrow E^{i}(M)=\operatorname{Ext}_{\mathbb{A}}^{i}(M, \mathbb{A}), i \geq 1$. En particulier, si le $\mathbb{A}$-module $M$ est de $\Lambda$-torsion, $E^{1}(M)$ n'est autre que l'adjoint d'Iwasawa ([Iw], 1.3), d'habitude noté $\alpha(M)$, muni d'une action supplémentaire de $H=\operatorname{Gal}\left(K_{\infty} / k_{\infty}\right)$. Remarquons que si $M$ est un $\mathbb{A}$-module à gauche, les $E^{i}(M)$ sont naturellement des $\mathbb{A}$-modules à droite, mais l'involution $\sigma \mapsto \sigma^{-1}$ dans les algèbres de groupes permet de récupérer des modules à gauche, qu'on notera $E^{i}(M)^{\sharp}$. Rappelons aussi que si $M$ et $N$ sont deux 
modules galoisiens à gauche, $\operatorname{Hom}(M, N)$ est muni d'une action galoisienne à gauche définie par ${ }^{\sigma} f(m)=\sigma\left(f\left(\sigma^{-1}(m)\right)\right)$.

3.1. Le module $E^{1}\left(X_{\infty}\right)$ :

Les modules adjoints associés aux divers modules $X_{\infty}, Y_{\infty}, Z_{\infty}, z_{\infty}$ (notations des $\S \S 1.1$ et 1.2) et à leurs résolutions respectives prennent place dans le diagramme commutatif exact suivant :

\subsubsection{Lemme :}

Avec les notations des $\S \S 1.1$ et 1.2 on a un diagramme commutatif exact de $\mathbb{A}$-modules (qui dépend de $d_{\infty}$ ):

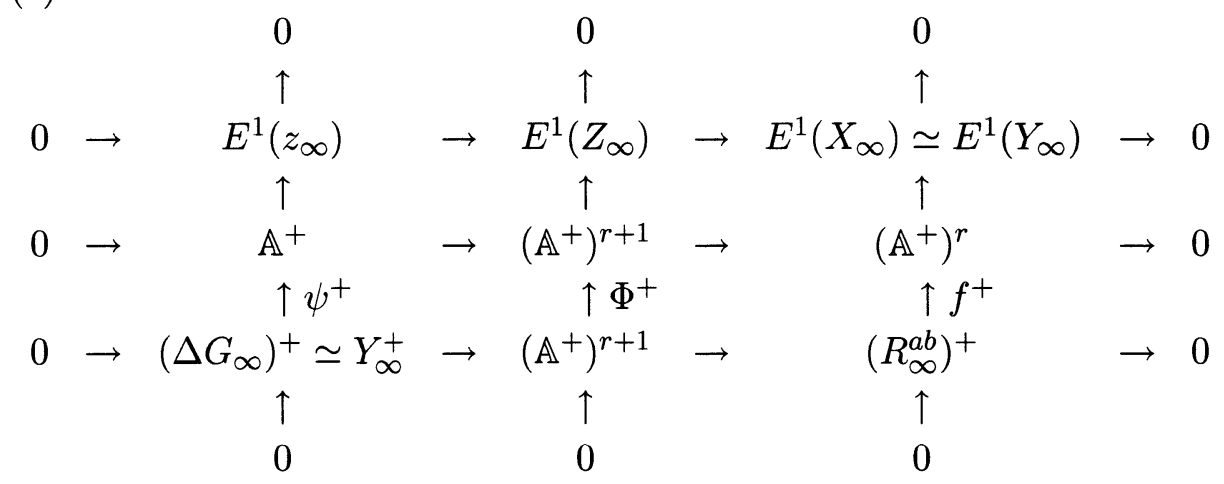

\section{Preuve :}

En mettant ensemble les diagrammes (2) et (3) de la section 1, on obtient un diagramme commutatif exact

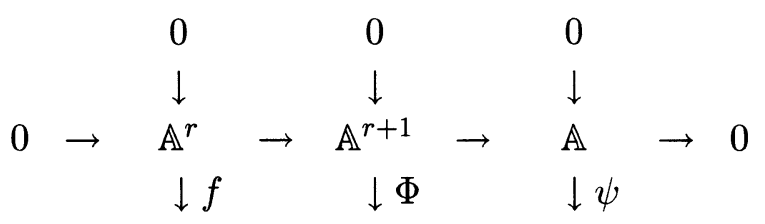

$$
\begin{aligned}
& 0 \rightarrow R_{\infty}^{a b} \stackrel{g}{\rightarrow} \mathbb{A}^{r+1} \stackrel{h}{\rightarrow} \Delta G_{\infty} \rightarrow 0
\end{aligned}
$$

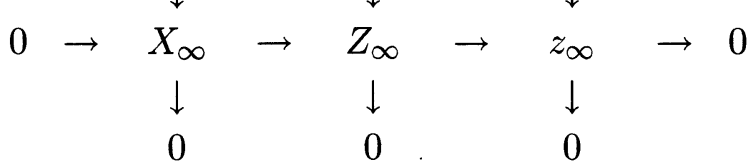

dont on déduit (4) en appliquant le foncteur $\operatorname{Hom}_{\mathbb{A}}(., \mathbb{A})$, la seule difficulté consistant à vérifier l'exactitude en certains points. Or, comme $\Gamma_{K}=\operatorname{Gal}\left(K_{\infty} / K\right)$ est ouvert dans $G_{\infty}=\operatorname{Gal}\left(K_{\infty} / k\right)$, on a ([J], lemme 2.3 ), pour tout $\mathbb{A}$-module $M$ et pour tout $i \geq 1$, un isomorphisme de $\Lambda$-modules $E_{\mathbb{A}}^{i}(M) \simeq E_{\Lambda}^{i}(M)$. Il en résulte en particulier que $E_{\mathbb{A}}^{i}\left(\mathbb{Z}_{p}\right)=0$ 
pour $i \geq 2$ car $c d_{p} \Gamma_{K}=1$, donc $E_{\AA}^{i}\left(\Delta G_{\infty}\right)=0$ pour $i \geq 1$ (par définition de l'augmentation) et $E_{\AA}^{i}\left(R_{\infty}^{a b}\right)=0$ pour $i \geq 1$ (par la résolution de Lyndon). L'exactitude du diagramme (4) en découle immédiatement. Il reste seulement à vérifier les isomorphismes $Y_{\infty}^{+} \simeq\left(\Delta G_{\infty}\right)^{+}$et $E^{1}\left(X_{\infty}\right) \simeq E^{1}\left(Y_{\infty}\right)$, ce qui provient de la suite exacte $0 \rightarrow X_{\infty} \rightarrow Y_{\infty} \rightarrow \Delta G_{\infty} \rightarrow 0$.

La dernière ligne et la dernière colonne du diagramme (4) mises ensemble donnent une $\mathbb{A}^{+}$-résolution de $E^{1}\left(X_{\infty}\right)$ indépendante de $d_{\infty}$ :

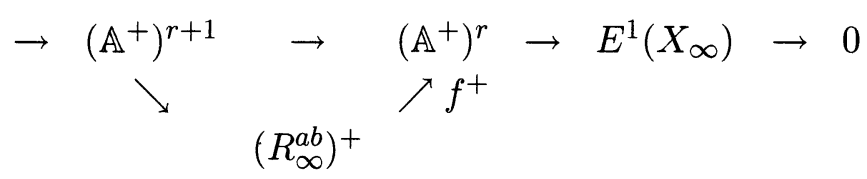

Le jeu va consister à calculer $F_{\mathbb{A}}\left(E^{1}\left(X_{\infty}\right)^{\sharp}\right)$ à partir de cette résolution. Par définition, l'idéal de Fitting sur $\mathbb{A}^{+}$de $E^{1}\left(X_{\infty}\right)$ est engendré par les sous-déterminants $(r \times r)$ extraits de la matrice (avec un abus de notation évident) Mat $\left(f^{+}\right)$, à $r$ lignes et $(r+1)$ colonnes. Or le carré commutatif en bas à droite du diagramme (4) permet de relier les matrices Mat $\left(f^{+}\right)$et Mat $\left(\Phi^{+}\right)$, qui s'obtiennent respectivement à partir de Mat $(f)$ (ici aussi, avec un abus de notation) et Mat $(\Phi)$ par transposition et inversion de l'action galoisienne. Retournons en détail à la construction de $\Phi$ dans le diagramme (3) du $\S 1.2$ :

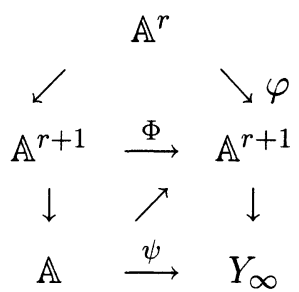

L'application $\Psi$ a été définie par $\Psi(1)=y_{\infty}=$ une pré-image de $d_{\infty}$. Le choix d'une section $\sigma: \mathbb{A} \rightarrow \mathbb{A}^{r+1}$ permet de définir $\widetilde{\Phi}=\Psi . \sigma$ par $\widetilde{\Phi}(1)=\widetilde{d}_{\infty}=$ une pré-image de $y_{\infty}$. De façon équivalente, $\widetilde{d}_{\infty}$ est une préimage de $d_{\infty}$ par l'application $\mathbb{A}^{r+1} \stackrel{h}{\rightarrow} \Delta G_{\infty}$ dans la fin de la résolution de Lyndon.

Or l'application $f$ prend place dans le carré commutatif :

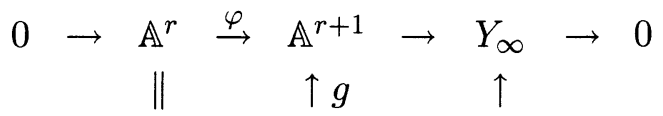

$$
\begin{aligned}
& 0 \rightarrow \mathbb{A}^{r} \stackrel{f}{\rightarrow} R_{\infty}^{a b} \quad \rightarrow \quad X_{\infty} \rightarrow 0 \quad \text { (diagramme (2) de 1.1.2) } \\
& \uparrow \uparrow \\
& 0 \quad 0
\end{aligned}
$$


où la flèche verticale du milieu $g$ est le début de la résolution de Lyndon. Il s'ensuit que le choix de la section $\sigma$ précédente permet d'écrire la matrice $(r+1) \times(r+1)$ Mat $(\Phi)$ sous la forme :

$$
\operatorname{Mat}(\Phi)=(\operatorname{Mat}(\varphi) \mid \operatorname{Mat}(\widetilde{\Psi}))
$$

où Mat $(\varphi)$ est une matrice $(r+1) \times r$ formée de vecteurs-colonnes engendrant $I m f$ et Mat $(\widetilde{\Phi})$ est une matrice $(r+1) \times 1$ représentant le vecteurcolonne $\widetilde{d}_{\infty}$. Par transposition et inversion galoisienne, on peut écrire Mat $\left(\Phi^{+}\right)$sous la forme

$$
\operatorname{Mat}\left(\Phi^{+}\right)=\left(\frac{\operatorname{Mat}\left(\varphi^{+}\right)}{\operatorname{Mat}\left(\widetilde{\Psi}^{+}\right)}\right)
$$

où Mat $\left(\varphi^{+}\right)$est formée de vecteurs-lignes engendrant $\operatorname{Im} f^{+}$et Mat $\left(\widetilde{\Psi}^{+}\right)$ est le vecteur-ligne obtenu à partir de $\widetilde{d}_{\infty}$.

Comme on l'a rappelé plus haut, $F_{\mathbb{A}^{+}}\left(E^{1}\left(X_{\infty}\right)\right)$ est engendré par les sous-déterminants $(r \times r)$ extraits de Mat $\left(\varphi^{+}\right)=$Mat $\left(f^{+}\right)$. Or un tel déterminant, au signe près, n'est autre qu'un certain dét $\left(\Phi^{+}\right)$où la dernière ligne a été choisie de la forme $(0 \ldots 1 \ldots 0)$. Si un tel choix est possible, i.e. s'il existe $\tilde{d}_{\infty}=\left(\begin{array}{c}0 \\ \vdots \\ 1 \\ \vdots \\ 0\end{array}\right)$ tel que $d_{\infty} \in \Delta G_{\infty}$ ne soit pas diviseur de zéro, la CPE3 nous dit que dét $(\Phi)=d_{\infty} \theta_{S}$. Nous sommes maintenant en mesure de démontrer le résultat principal de cette section, qui pourrait s'appeler CPE4. Pour tout idéal $I$ de $\mathbb{A}$, notons $I^{\sharp}$ l'idéal obtenu en inversant l'action galoisienne, i.e. en remplaçant tout $\sigma \in G_{\infty} \operatorname{par} \sigma^{-1}$. Alors :

\subsubsection{Théorème :}

Pour toute extension abélienne $K / k$ de corps totalement réels telle que $\mu_{p}=0$, on $a: F_{\mathbb{A}}\left(E^{1}\left(X_{\infty}\right)^{\sharp}\right)=\left(\Delta G_{\infty} \cdot \theta_{S}\right)^{\sharp} \quad(\mathrm{CPE} 4)$.

\section{Preuve :}

D'après les considérations précédentes, il suffit de construire des éléments particuliers $\widetilde{d}_{\infty}$. Ici encore, on peut découper suivant les idempotents associés à la non-p-partie de $G$ et se ramener immédiatement au cas où $G_{\infty}$ est un pro- $p$-groupe. Toutes les constructions du $\S 1.1$ peuvent alors se faire à l'intérieur de $G_{S}(k)(p)$, le groupe de Galois de la pro-p-extension 
$S$-ramifiée maximale de $k$, ce qui permet d'expliciter la résolution de Lyndon $([\mathrm{N} 1], \S 1)$. Nous refaisons une partie du calcul pour la commodité du lecteur :

Soit $G_{\infty} \simeq \widetilde{\Gamma} \times H$, avec $\widetilde{\Gamma}=\langle\widetilde{\gamma}\rangle$. Soit $\left\{\tau_{0}=\widetilde{\gamma}, \tau_{1}, \ldots, \tau_{s}\right\}$ un système minimal de générateurs de $G_{\infty}$. Le théorème de Burnside pour les pro- $p$ groupes permet de construire un système minimal de générateurs $\left\{\sigma_{0}=\right.$ $\left.\widehat{\gamma}, \sigma_{1}, \ldots, \sigma_{s}, \ldots, \sigma_{r}\right\}$ de $\mathcal{G}=G_{S}(k)(p)$ tel que, pour tout $0 \leq i \leq s$, l'image $\bar{\sigma}_{i}$ de $\sigma_{i}$ par la surjection canonique $\mathcal{G} \rightarrow G_{\infty}$ coïncide avec $\tau_{i}$. De plus, quitte à multiplier par exemple par $\widehat{\gamma}$, on peut supposer que les ordres de tous les $\bar{\sigma}_{j}$ sont divisibles par $p^{\infty}$, ce qui garantit, d'après une propriété connue des groupes profinis (voir e.g. [Ih], lemme 3.1), que les $\bar{\sigma}_{j}-1$, pour $0 \leq j \leq r$, ne sont pas des diviseurs de zéro dans $\mathbb{A}$. Réalisons alors $\mathcal{G}$ comme un quotient $\mathcal{F} / \mathcal{R}$ d'un pro-p-groupe libre sur $(r+1)$ générateurs $x_{0}, \ldots, x_{r}$ tels que $x_{j} \bmod \mathcal{R}=\sigma_{j}$. D'après la pro-p-version d'un théorème de Fox, l'idéal d'augmentation $\Delta \mathcal{F}$ est $\mathbb{Z}_{p} \llbracket \mathcal{F} \rrbracket$ libre sur les générateurs $\left(x_{j}-1\right)$. La suite exacte d'augmentation $0 \rightarrow \Delta \mathcal{F} \simeq \underset{j=0}{\oplus} \mathbb{Z}_{p} \llbracket \mathcal{F} \rrbracket\left(x_{j-1}\right) \rightarrow \mathbb{Z}_{p} \llbracket \mathcal{F} \rrbracket \rightarrow$ $\mathbb{Z}_{p} \rightarrow 0$ donne alors, par homologie, la résolution de Lyndon relative à $G_{\infty}$ :

$$
\begin{aligned}
0 \rightarrow R_{\infty}^{a b} \stackrel{g}{\longrightarrow} \oplus_{j=0}^{r} \mathbb{A} \varepsilon_{j} & \stackrel{h}{\longrightarrow} \Delta G_{\infty} \rightarrow 0 \\
\varepsilon_{j} & \mapsto\left(\bar{\sigma}_{j}-1\right)
\end{aligned}
$$

On a ainsi montré l'existence d'éléments particuliers $\widetilde{d}_{\infty}=\left(\begin{array}{c}0 \\ \vdots \\ 1 \\ \vdots \\ 0\end{array}\right) \in \mathbb{A}^{r+1}$ dont les images $d_{\infty} \in \Delta G_{\infty}$ ne sont pas diviseurs de zéro, et engendrent $\Delta G_{\infty}$. En se rappelant que l'opération $(.)^{\sharp}$ consiste à inverser l'action galoisienne et que deux matrices carrées transposées ont même déterminant, on en déduit le théorème.

\subsection{Autour du module $X_{\infty}$ :}

Les relations entre un $\mathbb{A}$-module $M$ et son adjoint $E^{1}(M)$ ne sont pas bien établies, sauf dans le cas où $p d_{\mathbb{A}} M \leq 1$. C'est pourquoi le théorème 3.1 .2 donne des renseignements plus précis sur $Y_{\infty}$ que sur $X_{\infty}$.

Commençons par $Y_{\infty}$. Nous noterons $F_{\AA}^{1}(M)$ le premier (après l'initial) idéal de Fitting d'un $\mathbb{A}$-module $M$.

\subsubsection{Proposition :}

Sous les hypothèses de 3.1.2, on a: $F_{\mathbb{A}}^{1}\left(Y_{\infty}\right)=\left(\Delta G_{\infty} \cdot \theta_{S}\right)^{\sharp}$. 


\section{Preuve :}

Le module $Y_{\infty}$ possède une résolution $0 \rightarrow \mathbb{A}^{r} \stackrel{\varphi}{\longrightarrow} \mathbb{A}^{r+1} \rightarrow Y_{\infty} \rightarrow 0$ d'où l'on tire, en appliquant le foncteur $(.)^{+}$, une résolution $\left(\mathbb{A}^{+}\right)^{r+1} \stackrel{\varphi^{+}}{\longrightarrow}$ $\left(\mathbb{A}^{+}\right)^{r} \rightarrow E^{1}\left(Y_{\infty}\right) \rightarrow 0$. Mais $E^{1}\left(Y_{\infty}\right) \simeq E^{1}\left(X_{\infty}\right)$ d'après 3.1.1, d'où $F_{\mathbb{A}}^{1}\left(Y_{\infty}\right)=F_{\mathbb{A}}\left(E^{1}\left(Y_{\infty}\right)^{\sharp}\right)=\left(\Delta G_{\infty} \cdot \theta_{S}\right)^{\sharp}$.

\section{Remarque :}

Si l'on suppose que $G_{\infty}$ est un pro-p-groupe (ce qui n'est pas une hypothèse restrictive), la matrice Mat $(\varphi)$ est une "matrice jacobienne" obtenue par "dérivation de Fox" à partir des $r$ relations du pro- $p$-groupe $G_{S}(k)(p)$ (pour des détails, voir [N1], $\S 4$ ). Malheureusement on ne connaît pas ces relations en général.

Passons maintenant à $X_{\infty}$. Nous nous placerons dans une situation particulière, déjà étudiée par de nombreux auteurs (voir e.g. les références données dans $[\mathrm{LF}])$ :

$$
H=\operatorname{Gal}\left(K_{\infty} / k_{\infty}\right)=P \times Q,
$$

où $P$ est le $p$-Sylow de $H$ et la non-p-partie $Q$ est supposée non triviale. Pour tout caractère $\xi \in \widehat{Q}=\operatorname{Hom}\left(Q, \overline{\mathbb{Q}}_{p}^{\times}\right.$) (qu'on peut aussi considérer comme un caractère de $H$ ), on notera $\varepsilon_{\xi}=\frac{1}{|Q|} \sum_{\sigma \in Q} \operatorname{Tr}(\xi(\sigma)) \sigma^{-1}$ et $e_{\xi}=\frac{1}{|H|} \sum_{\tau \in H} \operatorname{Tr}(\xi(\tau)) \tau^{-1}$ les idempotents primitifs associés. Comme d'habitude, en désignant par $\Lambda(\xi)$ l'extension de $\Lambda$ obtenue en ajoutant les valeurs de $\xi$, on a $\varepsilon_{\xi}(\mathbb{A}) \simeq \Lambda(\xi)[P]$.

\subsubsection{Proposition :}

Sous les hypothèses de 3.1.2, pour tout caractère non trivial $\xi$ de $\widehat{Q}$, on $a$ :

$$
F_{\Lambda(\xi)[P]}\left(\varepsilon_{\xi}\left(X_{\infty}\right)\right)=\Lambda(\xi)[P]^{\sharp} \cdot \varepsilon_{\xi^{-1}}\left(\Theta_{S}^{\bullet}\right)^{\sharp},
$$

où $\Theta_{S}^{\bullet} \in \mathbb{A}$ est défini par

$$
\Theta_{S}^{\bullet}=\sum_{\substack{\psi \in \hat{H} \\ \psi \neq 1}} G_{\psi, S}(\widetilde{\gamma}-1) e_{\psi} \quad \text { (notations de 2.2). }
$$

\section{Preuve :}

Comme $\xi \neq 1$, on a $\varepsilon_{\xi}\left(\Delta G_{\infty}\right)=\varepsilon_{\xi}(\mathbb{A})$ et la suite exacte $0 \rightarrow \varepsilon_{\xi}\left(X_{\infty}\right) \rightarrow$ $\varepsilon_{\xi}\left(Y_{\infty}\right) \rightarrow \varepsilon_{\xi}\left(\Delta G_{\infty}\right) \rightarrow 0$ se scinde pour donner une somme directe $\varepsilon_{\xi}\left(Y_{\infty}\right) \simeq \varepsilon_{\xi}\left(X_{\infty}\right) \oplus \Lambda(\xi)[P]$. La proposition 3.2.1 entraîne immédiatement 
que $F_{\Lambda(\xi)[P]}\left(\varepsilon_{\xi}\left(X_{\infty}\right)\right)=\Lambda(\xi)[P]^{\sharp} \cdot \varepsilon_{\xi^{-1}}\left(\theta_{S}\right)^{\sharp}$, et il reste seulement à calculer $\varepsilon_{\xi^{-1}}\left(\theta_{S}\right)^{\sharp}$. En posant $T=\widetilde{\gamma}-1$, on a :

$$
\theta_{S}=\sum_{\psi \in \widehat{H}} \frac{G_{\psi, S(T)}}{T^{\langle\psi, 1\rangle}} e_{\psi}=\frac{G_{1, S}(T)}{T} e+\Theta_{S}^{\bullet}
$$

dans $Q \mathbb{A}$ (voir formulaire 2.4). En notant $\nu$ la trace dans une algèbre de groupe, on peut écrire : $e_{1}=|H| \nu_{H}=|H| \nu_{P} . \nu_{Q}$, donc $e_{1} \varepsilon_{\xi^{-1}}=$ $|H| .|Q| \nu_{P} \varepsilon_{1} \varepsilon_{\xi^{-1}}$. Or $\varepsilon_{1} \varepsilon_{\xi^{-1}}=0$ pour $\xi \neq 1$, et donc $\varepsilon_{\xi^{-1}}\left(\theta_{S}\right)^{\sharp}=\varepsilon_{\xi^{-1}}\left(\Theta_{S}^{\bullet}\right)$.

\section{Remarque :}

Il apparaît clairement que les complications de l'idéal $F_{\mathbb{A}}\left(X_{\infty}\right)$ (qui n'est probablement pas principal) se concentrent au caractère trivial à cause $\mathrm{du}$ pôle de la fonction zêta $p$-adique.

\subsection{Groupes de cohomologie $p$-adique :}

En utilisant le fait que $p d_{\mathbb{A}}\left(Y_{\infty}\right) \leq 1, E^{1}\left(Y_{\infty}\right) \simeq E^{1}\left(X_{\infty}\right)$ et $X_{\infty}$ est de torsion, on peut appliquer les méthodes homotopiques (voir e.g. [J], thm. 1.6) pour montrer directement que $E^{1} E^{1}\left(X_{\infty}\right) \simeq X_{\infty}$. Alors :

\subsubsection{Proposition :}

Pour toute extension abélienne $K / k$ de corps totalement réels,

$$
\operatorname{Ann}_{\mathbb{A}}\left(X_{\infty}\right)=\operatorname{Ann}_{\mathbb{A}}\left(E^{1}\left(X_{\infty}\right)^{\sharp}\right)^{\sharp} .
$$

En particulier, si $\mu_{p}=0$, alors $\Delta G_{\infty} \cdot \theta_{S}$ annule $X_{\infty}$.

\section{Preuve :}

Posons $N=E^{1}\left(X_{\infty}\right)$ et soit $\left(\pi_{n}\right)$ une suite d'éléments de $\Lambda$, étrangers au diviseur du $\Lambda$-module $N$, tels que $\pi_{n+1}$ divise $\pi_{n}$ et $\pi_{n}$ tend vers zéro. D'après la théorie de l'adjoint d'Iwasawa, $E^{1}(N) \simeq \lim \left(N / \pi_{n} N\right)^{*}$, où (. . ${ }^{*}$ désigne le dual de Pontryagin. Notons $\Lambda_{n}=\mathbb{Z}_{p}\left[\mathrm{Gal}\left(K_{n} / k\right)\right]$. Il est facile de voir qu'un $\Lambda_{n}$-module $M$ a le même annulateur que son dual de Pontryagin modifié $\left(M^{*}\right)^{\sharp}$. La proposition en découle.

En général, un $\Lambda_{n}$-module n'a pas le même idéal de Fitting modifié que son dual, sauf si le $p$-Sylow de $G_{n}$ est cyclique (voir e.g. [Sn 2], propos. 2.8 ou $[\mathrm{MW}]$, appendix). On ne peut donc pas en général obtenir $F_{\mathbb{A}}\left(X_{\infty}\right)$ par la méthode précédente. On va plutôt faire un détour par la cohomologie $p$-adique. Rappelons que $G_{S}(L)$ désigne le groupe de Galois de l'extension $S$-ramifiée maximale d'un corps de nombres $L$, et donc que $G_{S}^{a b}(L)(p)=$ $X_{S}(L)$ (notations du $\S 1$ ). 


\subsubsection{Lemme :}

Soit $K_{\infty}=\bigcup_{n} K_{n}$. Notons $(.)^{*}$ le dual de Pontryagin. Alors, en supposant que tous les $K_{n}$ vérifient la conjecture de Leopoldt :

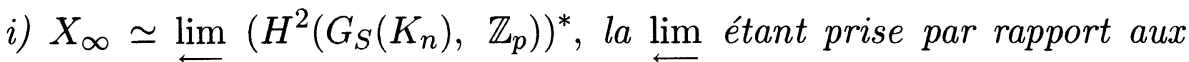
applications duales de la restriction.

ii) $E^{1}\left(X_{\infty}\right)^{\sharp} \simeq \underline{\lim } H^{2}\left(G_{S}\left(K_{n}\right), \mathbb{Z}_{p}\right)$, la lim étant prise par rapport à la corestriction.

\section{Preuve :}

La propriété i) est contenue implicitement dans [N2], § 2. Nous en redonnons une démonstration rapide pour la commodité du lecteur. Supposons que les $K_{n}$ vérifient la conjecture de Leopoldt, ce qui peut se traduire de deux façons :

- la surjection canonique $H^{2}\left(G_{S}\left(K_{n}\right), \mathbb{Z}_{p}\right)^{*} \rightarrow$ tor $_{\mathbb{Z}_{p}} X_{S}\left(K_{n}\right)$ est un isomorphisme

- avec les notations classiques de la théorie d'Iwasawa, les co-invariants $X_{\infty} / \omega_{n} X_{\infty}$ sont finis, et la suite exacte usuelle $0 \rightarrow X_{\infty} / \omega_{n} X_{\infty} \rightarrow X_{S}\left(K_{n}\right)$ $\rightarrow \mathbb{Z}_{p} \rightarrow 0$ montre alors que $X_{\infty} / \omega_{n} X_{\infty} \stackrel{\sim}{\rightarrow} \operatorname{tor}_{\mathbb{Z}_{p}} X_{S}\left(K_{n}\right)$. L'assertion i) est démontrée dans ce cas.

Pour montrer la propriété ii), on procède comme dans 3.3.1.

On va s'intéresser maintenant aux groupes de cohomologie $p$-adiques "tordus" à la Tate, plus précisément aux groupes $H^{2}\left(G_{S}\left(K_{n}\right), \mathbb{Z}_{p}(m)\right)$, pour $m \in \mathbb{Z}, m \equiv 0(\bmod 2)$. L'intérêt des groupes $H^{2}\left(G_{S}\left(K_{n}\right), \mathbb{Z}_{p}(m)\right)$, pour $m \geq 2$, est qu'ils sont canoniquement isomorphes aux groupes de $K$-théorie étale $K_{2 m-2}^{\text {ét }}\left(\mathcal{O}_{S, n}\right)$, où $\mathcal{O}_{S, n}$ est l'anneau des $S$-entiers de $K_{n}$ (Dwyer-Friedlander), et conjecturalement isomorphes aux tensorisés $K_{2 m-2}\left(\mathcal{O}_{S, n}\right) \otimes \mathbb{Z}_{p}$ (conjecture de Quillen-Lichtenbaum). On sait que les caractères de Chern $p$-adiques réalisent des surjections $K_{2 m-2}\left(\mathcal{O}_{S, n}\right) \otimes \mathbb{Z}_{p} \rightarrow$ $K_{2 m-2}^{\text {ét }}\left(\mathcal{O}_{S, n}\right)$ (Soulé, Dwyer-Friedlander) et que ces surjections sont scindées (B. Kahn). Il en résulte en particulier que les analogues "tordus" de la conjecture de Leopoldt sont vrais (voir e.g. [Sc]).

On notera $H_{S}^{2}\left(K_{\infty}, m\right):=\lim H^{2}\left(G_{S}\left(K_{n}\right), \mathbb{Z}_{p}(m)\right)$, la lim étant prise par rapport à la corestriction. Posons $K^{\prime}=K\left(\xi_{p}\right), K_{\infty}^{\prime}=\overleftarrow{K_{\infty}}\left(\xi_{p}\right)=K\left(\xi_{p \infty}\right)$, $\Delta=\operatorname{Gal}\left(K_{\infty}^{\prime} / K_{\infty}\right) \simeq \operatorname{Gal}\left(K^{\prime} / K\right)$ (ne pas confondre ce $\Delta$ avec la notation de l'idéal d'augmentation !). Comme $K_{\infty}^{\prime}$ contient $\xi_{p^{\infty}}, H_{S}^{2}\left(K_{\infty}^{\prime}, m\right)=$ $H_{S}^{2}\left(K_{\infty}^{\prime}, 0\right)(m)$, où $().(m)$ désigne la nouvelle action galoisienne définie par $\sigma . x=\chi_{\text {cyc }}(\sigma)^{m} \sigma(x)$. Comme $p \neq 2, \Delta$ est d'ordre premier à $p$, d'où $H_{S}^{2}\left(K_{\infty}, m\right)=H_{S}^{2}\left(K_{\infty}^{\prime}, m\right)^{\Delta}=\left(H_{S}^{2}\left(K_{\infty}^{\prime}, 0\right)(m)\right)^{\Delta}=\left(\varepsilon_{-m} H_{S}^{2}\left(K_{\infty}^{\prime}, 0\right)\right)(m)$, où $\varepsilon_{-m}$ est l'idempotent de $\Delta$ attaché à la puissance $\omega^{-m}$ du caractère de Teichmüller. 


\subsubsection{Théorème :}

Dans les hypothèses de 3.1.2, et pour tout $m \geq 2$, pair, on a :

$$
F_{\mathbb{A}}\left(H_{S}^{2}\left(K_{\infty}, m\right)\right)=t w_{m}\left(\Delta G_{\infty} . \theta_{S}\right),
$$

où two désigne l'automorphisme de $Q \mathbb{A}$ induit par le "twist"

$$
\sigma \mapsto \chi_{\text {cyc }}(\sigma)^{m} \sigma^{-1} \text {. }
$$

\section{Preuve :}

Soit $K_{\infty}^{\prime+}$ le sous-corps totalement réel maximal de $K_{\infty}^{\prime}$, et soit $\delta=$ Gal $\left(K_{\infty}^{\prime+} / K_{\infty}\right)$. Les considérations précédentes montrent que, pour tout $m$ pair, $H_{S}^{2}\left(K_{\infty}, m\right) \simeq H_{S}^{2}\left(K_{\infty}^{\prime+}, m\right)^{\delta} \simeq H_{S}^{2}\left(K_{\infty}^{\prime+}, m\right)_{\delta}$, donc l'idéal de Fitting de $H_{S}^{2}\left(K_{\infty}, m\right)$ s'obtient en prenant le co-invariant par $\delta$ de l'idéal de Fitting de $H_{S}^{2}\left(K_{\infty}^{\prime+}, m\right)$. On conclut en appliquant 3.1.2 et l'analogue tordu de $3.3 .2 \mathrm{ii})$.

On laisse au lecteur le soin de formuler un analogue de 3.2.2 en appliquant 3.3 .3 (cp. [LF], p. 286).

On va maintenant appliquer la co-descente aux CPE des $\S \S 2$ et 3 pour étudier, au niveau fini, l'annulation de certains modules galoisiens en termes de fonctions $L$.

\section{Sur la conjecture de Coates-Sinnott}

Les hypothèses sont les mêmes que depuis le début, c'est-à-dire que $K / k$ est une extension galoisienne de corps de nombres totalement réels, de groupe de Galois $G$. On note $S_{p}=S_{p}(K)$ l'ensemble des $p$-places de $K, R$ $=\operatorname{Ram}(K / k)$ l'ensemble des places de $K$ qui sont ramifiées dans $K / k$, et $S=S_{p} \cup R$.

\section{1. Éléments de Stickelberger :}

Dans cette sous-section, $F / k$ est une extension abélienne (non forcément réelle), de groupe $G$, et $S$ est un ensemble fini de places contenant $R$. On convient que toutes les fonctions $L$ sont relatives au corps de base $k$, qui ne figurera pas dans les notations. Pour tout $m \in \mathbb{N}$, l'élément de Stickelberger $\operatorname{Stick}_{S}^{(m)}(K / k)$ est l'unique élément de $\mathbb{Q}[G]$ tel que, pour tout caractère absolument irréductible $\chi$ de $G$, on ait : $\chi\left(\operatorname{Stick}_{S}^{(m)}(K / k)\right)=$ $L_{S}\left(1-m, \chi^{-1}\right)$, où $L_{S}$ est la fonction $L$ complexe privée des facteurs euleriens en $S$. Autrement dit, en notant $e_{\chi}$ les idempotents usuels :

$$
\forall m \in \mathbb{N}, \quad \operatorname{Stick}_{S}^{(m)}(K / k)=\sum_{\chi \in \widehat{G}} L_{S}\left(1-m, \chi^{-1}\right) e_{\chi} .
$$

On notera simplement $\operatorname{Stick}^{(m)}(K / k)$ au lieu de $\operatorname{Stick}_{R}^{(m)}(K / k)$. 
La conjecture de Coates-Sinnott ([CS]), modifiée par V. Snaith ([Sn1], p. 280), par analogie avec le théorème classique de Stickelberger, prédit le résultat d'annulation galoisienne suivant :

\subsection{Conjecture (CS) :}

Soit $K / k$ une extension abélienne de corps totalement réels, de groupe G. Pour tout $m \geq 2$, pair,

$$
\operatorname{Ann}_{\mathbb{Z}[G]}\left(\operatorname{tor}_{\mathbb{Z}} K_{2 m-1}(K)\right) . \operatorname{Stick}^{(m)}(K / k) \subseteq A n n_{\mathbb{Z}[G]}\left(K_{2 m-2}\left(\mathcal{O}_{K}\right)\right) .
$$

Notons que la conjecture contient en fait deux assertions :

- le premier membre appartient à l'algèbre de groupe entière $\mathbb{Z}[G]$

- il annule le module $K_{2 m-2}\left(\mathcal{O}_{K}\right)$.

Comme $K_{2 m-2}\left(\mathcal{O}_{K}\right)$ est fini, on peut l'annuler $p$-composante par $p$ composante, et c'est ce qu'on se propose de faire par co-descente à partir de 3.3.3. Notons ici que, contrairement à l'habitude dans ce type de problèmes $p$-adique, nous ne supposons pas que les extensions $K$ et $k_{\infty}$ sont linéairement disjointes sur $k$. Ce degré de généralité est nécessaire pour globaliser le résultat (voir 4.4 ci-dessous).

\subsection{Théorème :}

Si $p \neq 2$ et $\mu_{p}=0$, alors pour tout $m \geq 2$, pair :

$$
\begin{aligned}
F_{\mathbb{Z}_{p}[G]}\left(H ^ { 2 } \left(G_{S}(K),\right.\right. & \left.\mathbb{Z}_{p}(m)\right) \\
& =F_{\mathbb{Z}_{p}[G]}\left(\operatorname{tor}_{\mathbb{Z}_{p}} H^{1}\left(G_{S}(K), \mathbb{Z}_{p}(m)\right)\right) \cdot \operatorname{Stick}^{(m)}(K / k)
\end{aligned}
$$

\section{Preuve :}

Comme $c d_{p} G_{S}(k) \leq 2(\operatorname{car} p \neq 2)$, on sait que les $H^{2}($.$) vérifient la co-$ descente galoisienne, i.e. pour toute extension galoisienne $S$-ramifiée $M / L$, de groupe de Galois $J$, on a $H^{2}\left(G_{S}(L), \mathbb{Z}_{p}(m)\right) \simeq H^{2}\left(G_{S}(M), \mathbb{Z}_{p}(m)\right)_{J}$. Il en résulte que le Fitting de $H^{2}\left(G_{S}(K), \mathbb{Z}_{p}(m)\right)$ est l'image du Fitting de $H_{S}^{2}\left(K_{\infty}, m\right)$ (qui est donné par 3.3.3) par la surjection canonique $\mathbb{Z}_{p}\left[\left[G_{\infty}\right]\right] \rightarrow \mathbb{Z}_{p}[G], z \mapsto \bar{z}$. On garde les notations des $\S \S 2$ et 3 . En particulier, $\lambda_{S}$ et $\theta_{S}$ sont les pseudo-mesures de Deligne-Ribet de $X_{S}(k)$ et $G_{\infty}$. Pour déterminer les éléments de la forme $t w_{m}(\sigma-1) . \theta_{S}$, avec $\sigma \in G_{\infty}$, il suffit de calculer les valeurs des caractères $\chi$ de $G$ appliqués à ces éléments. On peut considérer $\chi$ comme un caractère de $G_{\infty}$. Alors, par définition du "twist" (3.3.3) et de l'intégration par rapport à une pseudo-mesure 
([Se], 1.11), on a :

$$
\begin{aligned}
\chi\left(t w_{m}(\sigma-1) \cdot \theta_{S}\right) & =\left\langle\chi^{-1} \chi_{\mathrm{cyc}}^{m}, \theta_{S}\right\rangle \cdot\left(\chi^{-1} \chi_{\mathrm{cyc}}^{m}(\sigma)-1\right) \\
& =\left\langle\chi^{-1} \chi_{\mathrm{cyc}}^{m}, \lambda_{S}\right\rangle \cdot\left(\chi^{-1} \chi_{\mathrm{cyc}}^{m}(\sigma)-1\right) .
\end{aligned}
$$

Rappelons que le caractère cyclotomique s'écrit $\chi_{\mathrm{cyc}}=\omega \kappa$. D'après [Se], 3.5 , pour tout caractère $\varphi$ d'ordre fini de $X_{S}(k)$,on a :

$$
\left\langle\varphi \kappa^{m}, \lambda_{S}\right\rangle=L_{S}\left(1-m, \varphi \omega^{-m}\right),
$$

d'où finalement :

$$
\chi\left(t w_{m}(\sigma-1) \theta_{S}\right)=\left(\chi^{-1} \chi_{\mathrm{cyc}}^{m}(\sigma)-1\right) L_{S}\left(1-m, \chi^{-1}\right) .
$$

Cela montre que $F_{\mathbb{Z}_{p}[G]}\left(H^{2}\left(G_{S}(K), \mathbb{Z}_{p}(m)\right)\right.$ est l'idéal de $\mathbb{Z}_{p}[G]$ engendré par les éléments $\left(\chi_{\text {cyc }}^{m}(\bar{\sigma}) \bar{\sigma}^{-1}-1\right) L_{S}\left(1-m, \chi^{-1}\right), \bar{\sigma}$ parcourant $G$. Or :

- d'une part, il est bien connu (voir e.g. $[\mathrm{KNF}]$, lemma 2.2) qu'en prenant les co-invariants de $\mathbb{Z}_{p}(m)$ par $\operatorname{Gal}\left(K_{\infty} / K\right)$, on obtient $H^{0}\left(G_{S}(K)\right.$, $\left.\mathbb{Q}_{p} / \mathbb{Z}_{p}(m)\right)$, qui est lui-même isomorphe à tor $\mathbb{Z}_{p}\left(H^{1}\left(G_{S}(K), \mathbb{Z}_{p}(m)\right)\right)$. Le $\mathbb{Z}_{p}[G]$-Fitting de ce dernier module est donc l'image dans $\mathbb{Z}_{p}[G]$ du $\mathbb{Z}_{p}\left[\left[G_{\infty}\right]\right]$-Fitting de $\mathbb{Z}_{p}(m)$. Comme le $\mathbb{Z}_{p}\left[\left[G_{\infty}\right]\right]$-Fitting de $\mathbb{Z}_{p}$ est $\Delta G_{\infty}$, on vérifie immédiatement que $F_{\mathbb{Z}_{p}[G]}\left(\operatorname{tor}_{\mathbb{Z}_{p}} H^{1}\left(G_{S}(K), \mathbb{Z}_{p}(m)\right)\right)$ est engendré par les éléments $\left(\chi_{\text {cyc }}^{m}(\bar{\sigma}) \bar{\sigma}^{-1}-1\right), \bar{\sigma} \in G$.

- d'autre part,

$$
L_{S}\left(1-m, \chi^{-1}\right)=L_{R}\left(1-m, \chi^{-1}\right) \prod_{v \in S \backslash R}\left(1-\chi^{-1}(v)(N v)^{m-1}\right),
$$

où $R=\operatorname{Ram}(K / k)$. Pour $m \geq 2$ et $v \mid p$, le facteur eulerien $\left(1-(N v)^{m-1}\right.$ Frob $_{v}^{-1}$ ) est clairement inversible dans $\mathbb{Z}_{p}[G]$ (il suffit de développer formellement l'inverse en série entière et de regrouper suivant les puissances du Frobenius).

Le théorème 4.3 est ainsi démontré.

\section{Remarque :}

Dans une tour d'extensions abéliennes finies $S$-ramifiées $M / L / k$, on sait ([T], p. 86) que l'image $\operatorname{de~} \operatorname{Stick}_{S}^{(m)}(M / k)$ dans $\mathbb{Q}_{p}[\operatorname{Gal}(L / k)]$ coïncide avec $\operatorname{Stick}_{S}^{(m)}(L / k)$ et donc que la limite projective $\operatorname{Stick}_{S}^{(m)}\left(K_{\infty}\right):=$ $\lim \operatorname{Stick}_{S}^{(m)}\left(K_{n} / k\right)$ existe. Les calculs de 4.3 montrent en fait que, pour tout $m \geq 1$, les idéaux $t w_{m}\left(\Delta G_{\infty} \cdot \theta_{S}\right)$ et $t w_{m}\left(\Delta G_{\infty} \cdot \operatorname{Stick}_{S}^{(m)}\left(K_{\infty}\right)\right)$ coïncident : si $m$ est impair et $m \geq 3$, les deux idéaux sont nuls et il n'y a rien à montrer ; si $m$ est pair, $m \geq 2$, c'est le calcul fait dans la démonstration de 4.3 ; et ce calcul reste valable pour $m=1$. 
Revenons à la conjecture (CS). Pour $p \neq 2$ et $i=1,2$, comme on l'a rappelé plus haut, les caractères de Chern $p$-adiques induisent des homomorphismes surjectifs scindés $K_{2 m-i}\left(\mathcal{O}_{K}^{S}\right) \otimes \mathbb{Z}_{p} \rightarrow H^{i}\left(G_{S}(K), \mathbb{Z}_{p}(m)\right)$ (pour tout $S \supseteq S_{p}$ ), qui sont conjecturalement des isomorphismes. De plus, la suite exacte de localisation en $K$-théorie montre que, pour tout $m \geq 2$, $K_{2 m-1}\left(\mathcal{O}_{K}\right) \simeq K_{2 m-1}(K)$ et $K_{2 m-2}\left(\mathcal{O}_{K}\right) \otimes \mathbb{Z}_{p} \simeq K_{2 m-2}\left(\mathcal{O}_{K}^{S_{p}}\right) \otimes \mathbb{Z}_{p} \hookrightarrow$ $K_{2 m-2}\left(\mathcal{O}_{K}^{S}\right) \otimes \mathbb{Z}_{p}$. Idem pour la $K$-théorie étale ou la cohomologie étale. Posons $K_{2 m-i}^{c o h}\left(\mathcal{O}_{K}\right):=\oplus_{p \neq 2} H^{i}\left(G_{S_{p}}(K), \mathbb{Z}_{p}(m)\right)$, considéré comme sousmodule de $K_{2 m-i}\left(\mathcal{O}_{K}\right)$. Notons $\mathbb{Z}^{\prime}=\mathbb{Z}[1 / 2]$.

Nous pouvons maintenant montrer, à la composante 2-primaire près, la version cohomologique (affinée) de la conjecture de Coates-Sinnott :

\subsection{Théorème :}

Soit $K / k$ une extension abélienne de corps totalement réels, de groupe de Galois $G$, vérifiant $\mu_{p}=0$ pour tout $p \neq 2$. Pour tout entier pair $m \geq 2$, on $a$ :

$$
\begin{array}{r}
F_{\mathbb{Z}^{\prime}[G]}\left(\operatorname{tor}_{\mathbb{Z}^{\prime}} K_{2 m-1}^{c o h}\left(\mathcal{O}_{K}\right) \otimes \mathbb{Z}^{\prime}\right) . \operatorname{Stick}^{(m)}(K / k) \\
\subseteq A n n_{\mathbb{Z}^{\prime}[G]}\left(K_{2 m-2}^{c o h}\left(\mathcal{O}_{K}\right) \otimes \mathbb{Z}^{\prime}\right)
\end{array}
$$

\section{Preuve :}

La suite exacte de localisation en cohomologie étale, ou le théorème de comparaison entre $G_{S}$ et $G_{S_{p}}$ ([NSW], X,5), montrent immédiatement que $H^{2}\left(G_{S_{p}}(K), \mathbb{Z}_{p}(m)\right) \subseteq H^{2}\left(G_{S}(K), \mathbb{Z}_{p}(m)\right)$. Par ailleurs, on sait que l'idéal de Fitting d'un module est contenu dans son annulateur. Il suffit alors d'appliquer le théorème 4.3 .

\section{Remarques :}

i) Dans la situation où $G=P \times Q$ (notations de 3.2.2), on peut déterminer, par co-descente à partir de 3.3.3, les idéaux de Fitting des $\xi$-composantes de $K_{2 m-2}^{c o h}\left(\mathcal{O}_{K}\right), \xi$ parcourant les caractères non triviaux de $Q$, retrouvant ainsi le résultat principal de $[\mathrm{LF}]$. Nous n'entrerons pas dans les détails.

ii) Pour $m \geq 2$, impair, l'énoncé 4.4 est trivial. Pour avoir un énoncé significatif, il faut recourir, du côté analytique, aux "valeurs spéciales" (c'est-à-dire les valeurs des dérivées) des fonctions $L$, et du côté algébrique, aux "éléments spéciaux". Dans le cas absolument abélien (i.e. $k=\mathbb{Q}$ ), ces éléments spéciaux sont des réalisations $p$-adiques et archimédiennes des éléments de Beilinson en $K$-théorie ([KNF], [BN], [BG1], [HK1], etc $\ldots$ ), mais pour $k \neq \mathbb{Q}$, leur existence est encore conjecturale (voir les développements autour des conjectures de Stark). 
iii) V. Voevodsky a annoncé récemment la démonstration de la conjecture de Bloch-Kato sur le lien entre la $K$-théorie de Milnor mod $p$ et la cohomologie galoisienne. Or cette conjecture entraîne celle de Quillen-Lichtenbaum (voir e.g. [Ro-W], dont les arguments restent valables pour $p \neq 2$ ). Dans 4.4 , on pourrait donc remplacer $K_{2 m-2}^{c o h}$ par $K_{2 m-2}$.

Récemment, D. Barsky a annoncé la démonstration de la nullité de $\mu_{p}$ pour $p \neq 2$ et tout corps totalement réel ([B]). L'énoncé de 4.4 deviendrait donc inconditionnel.

\section{Sur la conjecture de Brumer}

Dans cette section, on va faire un léger changement de notation par rapport au début. On désigne toujours par $K / k$ une extension abélienne de corps totalement réels. On suppose en outre que $K$ est le sous-corps totalement réel maximal d'un corps $F$ qui est $\mathrm{CM}$ et qui est une extension abélienne de $k$. Le changement de notation concerne $G$, qui désignera désormais le groupe de Galois de $F / k$. La conjecture de Brumer pour $F / k$ est une généralisation directe du théorème de Stickelberger pour $F / \mathbb{Q}$. Elle s'énonce ainsi :

\subsection{Conjecture (B) :}

Avec les notations précédentes,

$$
\operatorname{Ann}_{\mathbb{Z}[G]}(\mu(F)) . \operatorname{Stick}^{(1)}(F / k) \subseteq \operatorname{Ann}_{\mathbb{Z}[G]}(C \ell(F)),
$$

où $\mu(F)$ et $C \ell(F)$ sont respectivement le groupe des racines de l'unité et le groupe des classes d'idéaux de $F$.

Notons l'analogie avec la conjecture (CS), puisque $\mu(F)=\operatorname{tor}_{\mathbb{Z}} K_{1}\left(\mathcal{O}_{F}\right)$ et $C \ell(F)=\operatorname{tor}_{\mathbb{Z}} K_{0}\left(\mathcal{O}_{F}\right)$. Pour tout $G$-module $M$, soit $M^{+}$le sous-module fixé par la conjugaison complexe, et soit $M^{-}=M / M^{+}$. Il est bien connu (voir e.g. [G1], p. 517) que dans la conjecture (B), l'annulation de la partie $C \ell(F)^{+}$est automatique. Comme on néglige la partie 2-primaire, il reste seulement à vérifier l'annulation de la partie $C \ell(F)^{-}$. Pour cela, on peut procéder $p$-composante par $p$-composante et utiliser la CPE comme dans la démonstration de la conjecture (CS). Malheureusement, on verra que le passage du $p$-adique au global se heurtera au phénomène des "zéros triviaux" des fonctions $L_{p}$ (voir aussi les commentaires au début de [G1]).

\subsection{Théorème :}

Avec les notations précédentes, fixons en outre un nombre premier impair $p$ tel que $\mu_{p}=0$ pour la $\mathbb{Z}_{p}$-extension cyclotomique de $K=F^{+}$. Soit $S$ un ensemble fini équivariant de places de $F$ contenant $S_{p}(F) \cup \operatorname{Ram}(F / k)$. Alors le p-groupe de classes $A(F)$ est tué par

$$
\operatorname{Ann}_{\mathbb{Z}_{p}[G]}\left(\mu_{p \infty}(F)\right) . \operatorname{Stick}_{S}^{(1)}(F / k) .
$$




\section{Preuve :}

On garde les notations $X_{\infty}^{S}$ et $X_{\infty}^{S_{p}}$ de l'introduction, et l'on pose $A_{\infty}=$ $\lim A\left(F_{n}\right)$. On peut supposer sans perte de généralité que $F$ contient une $\overrightarrow{\text { racine }} p^{\text {ième }}$ primitive de l'unité. Par la théorie de Kummer, il est bien connu $([\mathrm{Iw}],[\mathrm{NSW}] \ldots)$ que $X_{\infty}^{S_{p}}\left(F^{+}\right)=X_{\infty}^{S_{p}}(F)^{+} \simeq \operatorname{Hom}\left(A_{\infty}^{-}, \mu_{p^{\infty}}\right)$, ce que l'on peut écrire :

$$
X_{\infty}^{S_{p}}(K)(-1) \simeq \operatorname{Hom}\left(A_{\infty}^{-}, \mathbb{Q}_{p} / \mathbb{Z}_{p}\right) .
$$

D'après 3.3.1, $\left(\Delta G_{\infty} \cdot \theta_{S}\right)(1)$, où $G_{\infty}=\operatorname{Gal}\left(K_{\infty} / k\right)$, annule $X_{\infty}^{S}(K)(-1)$, donc aussi $X_{\infty}^{S_{p}}(K)(-1)$. Par suite, l'image de cet idéal dans $\mathbb{Z}_{p}[\mathrm{Gal}(K / k)]$, disons $I$, annule $X_{\infty}^{S^{p}}(K)(-1)_{\Gamma} \simeq \operatorname{Hom}\left(\left(A_{\infty}^{-}\right)^{\Gamma}, \mathbb{Q}_{p} / \mathbb{Z}_{p}\right)$, où $\Gamma=$ Gal $\left(F_{\infty} / F\right)$. Il en résulte que $I^{\sharp}$ annule $\left(A_{\infty}^{-}\right)^{\Gamma}$, donc aussi $A(F)^{-}$ (dont il est bien connu qu'il s'identifie à un sous-module de $\left(A_{\infty}^{-}\right)^{\Gamma}$ ). Or $I^{\sharp}$ n'est autre que l'image dans $\mathbb{Z}_{p}[\mathrm{Gal}(K / k)]$ de $t w_{1}\left(\Delta G_{\infty} \cdot \theta_{S}\right)=$ $t w_{1}\left(\Delta G_{\infty} . \operatorname{Stick}_{S}^{(1)}\left(K_{\infty}\right)\right.$ ) (voir la remarque suivant le théorème 4.3). L'annulation de $A(F)^{+}$étant automatique, on a bien le résultat cherché.

Pour passer du $p$-adique au global, il faudrait avoir, comme dans 4.3, un énoncé $p$-adique ne dépendant que de $R=\operatorname{Ram}(F / k)$. Mais ici, les facteurs euleriens du type $\left(1-(N v)^{-1} \operatorname{Frob}_{v}^{-1}\right)$, pour les places $v$ divisant $p$ et non ramifiées dans $F / k$, ne sont pas inversibles dans $\mathbb{Z}_{p}[G]$. On peut se débarrasser de ces facteurs indésirables en ajoutant des hypothèses sur la ramification, par exemple, comme dans [G1], la condition :

$(G)$ Pour toute place $v \mid p$, le groupe de décomposition $G_{v}$ contient la conjugaison complexe.

Alors :

\subsection{Corollaire :}

Si $p$ vérifie en outre la condition $(G)$,

$$
A(F) \text { est tué par } \operatorname{Ann}_{\mathbb{Z}_{p}[G]}\left(\mu_{p^{\infty}}(F)\right) . \operatorname{Stick}_{R}^{(1)}(F / k),
$$

$c$-à-d. que la version $p$-adique de la conjecture (B) est vraie.

Mais ce résultat n'est toujours pas satisfaisant, puisque la condition $(G)$ ne peut pas être globalisée. Nous espérons revenir sur ce problème dans un travail ultérieur.

\section{Bibliographie}

[B] D. BARSKY, Sur la nullité du $\mu$-invariant d'Iwasawa des corps totalement réels, prépublication (2005).

[BG1] D. Burns \& C. Greither, On the Equivariant Tamagawa Number Conjecture for Tate motives. Invent. Math. 153 (2003), no. 2, 303-359. 
[BG2] D. BuRNS \& C. GReITHER, Equivariant Weierstrass Preparation and values of L-functions at negative integers. Doc. Math. (2003), Extra Vol., 157-185.

[BN] D. Benois \& T. NguYen QuAng Do. Les nombres de Tamagawa locaux et la conjecture de Bloch et Kato pour les motifs $\mathbb{Q}(m)$ sur un corps abélien. Ann. Sci. ENS 35 (2002), 641-672.

[CS] J. COATES \& W. SinnotT, An analogue of Stickelberger's theorem for the higher K-groups. Invent. Math. 24 (1974), 149-161.

[DR] P. DELIGNE \& K. RIBET, Values of abelian L-functions at negative integers. Invent. Math 59 (1980), 227-286.

[G1] C. GREITHER, The structure of some minus class groups, and Chinburg's third conjecture for abelian fields. Math. Zeit. 229 (1998), 107-136.

[G2] C. Greither, Some cases of Brumer's conjecture. Math. Zeit. 233 (2000), 515-534.

[G3] C. Greither, Computing Fitting ideals of Iwasawa modules. Math. Z. 246 (2004), no. $4,733-767$.

[HK1] A. HUBER \& G. KINGS, Bloch-Kato Conjecture and Main Conjecture of Iwasawa theory for Dirichlet characters. Duke Math. J. 119 (2003), no. 3, 393-464.

[HK2] A. Huber \& G. KIngs, Equivariant Bloch-Kato Conjecture and non abelian Iwasawa Main Conjecture. ICM 2002, vol. II, 149-162.

[Ih] Y. IHARA, On Galois representations arising from towers of coverings of $\mathbb{P}^{1} \backslash\{0,1, \infty\}$. Invent. Math. 86 (1986), 427-459.

[Iw] K. IWASAWA, On $\mathbb{Z}_{\ell}$-extensions of algebraic number fields. Annals of Math. 98 (1973), 246-326.

[J] U. JANNSEN, Iwasawa modules up to isomorphism. Adv. Studies in Pure Math. 17 (1989), 171-207.

[K] K. KATO, Lectures on the approach to Iwasawa theory for Hasse-Weil L-functions via $B_{\mathrm{dR}}$. I. Arithmetic algebraic geometry (Trento, 1991). 50-163, Lecture Notes in Math., 1553, Springer, Berlin, 1993.

[K1] M. KurIHARA, Iwasawa theory and Fitting ideals. J. Reine Angew. Math. 561 (2003), 39-86.

[K2] M. KURIHARA, On the structure of ideal class groups of CM fields. Doc. Math. (2003), Extra Vol., 539-563.

[KNF] M. Kolster, T. NguYen QuAng Do \& V. Fleckinger, Twisted S-units, p-adic class number formulas, and the Lichtenbaum conjectures. Duke Math. J. 84 (1996), no. 3, 679-717.

[LF] M. LE FLoC'H, On Fitting ideals of certain étale K-groups. K-Theory 27 (2002), 281292.

[MW] B. Mazur \& A. Wiles, Class fields of abelian extensions of $\mathbb{Q}$. Invent. Math. 76 (1984), 179-330.

[N1] T. NGUYen QuAng Do, Formations de classes et modules d'Iwasawa. Dans "Number Theory Noordwijkerhout", Springer LNM 1068 (1984), 167-185.

[N2] T. NGUYen QuANG Do, Sur la $\mathbb{Z}_{p}$-torsion de certains modules galoisiens. Ann. Inst. Fourier 36 (1986), no. 2, 27-46.

[N3] T. NguYen QuAng Do, Analogues supérieurs du noyau sauvage. J. Théorie des Nombres Bordeaux 4 (1992), 263-271.

[N4] T. NGUYen QuANG Do, Quelques applications de la Conjecture Principale Equivariante, lettre à M. Kurihara $(15 / 02 / 02)$.

[NSW] J. Neukirch. A. Schmidt \& K. Wingberg, Cohomology of Number Fields. Grundlehren 323, Springer, 2000.

[R] K. RIBET, Report on p-adic L-functions over totally real fields. Astérisque 61 (1979), 177-192.

[RW1] J. RITTER \& A. WeISS, The Lifted Root Number Conjecture and Iwasawa theory. Memoirs AMS 157/748 (2002).

[RW2] J. RitTER \& A. WeISS, Towards equivariant Iwasawa theory. Manuscripta Math. 109 (2002), 131-146. 
[Ro-W] J. Rognes \& C.A. WeIBEL, Two-primary algebraic K-theory of rings of integers in number fields. J. AMS (1) 13 (2000), 1-54.

[Sc] P. SCHNEIDER, Über gewisse Galoiscohomologiegruppen. Math. Zeit 168 (1979), 181-205.

[Se] J.-P. SERRE, Sur le résidu de la fonction zêta p-adique d'un corps de nombres. CRAS Paris 287, A (1978), 183-188.

[Sn1] V. SNAITH, “Algebraic K-groups as Galois modules”. Birkhauser, Progress in Math. 206 (2002).

[Sn2] V. SNAITH, Relative $K_{0}$, Fitting ideals and the Stickelberger phenomena, preprint (2002).

[T] J. TATE, "Les conjectures de Stark sur les fonctions $L$ d'Artin en $s=0$ ". Birkhauser, Progress in Math. 47 (1984).

[W1] A. WILES, The Iwasawa conjecture for totally real fields. Annals of Math. 141 (1990), 493-540.

[W2] A. WILES, On a conjecture of Brumer. Annals of Math. 131 (1990), 555-565.

Thong NGUYEN QUANG Do

UMR 6623 CNRS

Université de Franche-Comté

16, Route de Gray

25030 Besançon Cedex - France

E-mail : nguyen@math.univ-fcomte.fr 